\title{
Deformación frágil en rocas del Mioceno de la región Morelia-Cuitzeo, Michoacán: implicaciones en el sistema geotérmico local
}

\author{
Alberto Vásquez-Serrano ${ }^{1 *}$, Elizabeth Rangel-Granados ${ }^{1}$, Víctor Hugo Garduño-Monroy ${ }^{2}$, \\ Gerardo Bermejo Santoyo ${ }^{2}$ y Adrián Jiménez-Haro ${ }^{2}$
}

\author{
${ }^{1}$ Departamento de Procesos Litosféricos, Instituto de Geología. Universidad Nacional Autónoma de México, \\ Ciudad Universitaria, Ciudad de México, México. \\ ${ }^{2}$ Instituto de Investigaciones Metalúrgicas, Universidad Michoacana de San Nicolás Hidalgo, \\ Cuerpo Académico Consolidado de Ciencias de la Tierra, Ciudad Universitaria, Morelia, Michoacán, México. \\ *alberto-vasquez@ciencias.unam.mx
}

\section{RESUMEN}

La deformación frágil se relaciona con la presencia de fallas y fracturas que afectan a la corteza superior de la Tierra. La distribución que tiene la deformación frágil y el arreglo que presentan las fallas y fracturas son variables poco conocidas, a pesar de que tienen una gran influencia en la generación de permeabilidad y son importantes para entender fenómenos como la deformación localizada. En este trabajo se explora la distribución de la deformación frágil y el arreglo de las fracturas que afectan a rocas andesíticas del Mioceno en el área de Morelia-Cuitzeo mediante un análisis cuantitativo de la deformación a escala mesoscópica. Las rocas miocénicas del área de estudio están afectadas por dos familias de fracturas (F1 y F2) relacionadas con dos eventos de deformación frágil (D1 y D2) ocurridos entre el Mioceno y el Holoceno. Las fracturas F1 tienen un rumbo preferencial NNW-SSE y acomodan una extensión ENE-WSW de $\sim 2.5 \%$. Por otro lado, las fracturas F2 cortan a las fracturas F1, tienen un rumbo ENE-WSW y se relacionan con una extensión $\sim \mathrm{N}-\mathrm{S}$ de $6.8 \%$. Ambas familias de fracturas tienen un arreglo de fracturas agrupadas y una intensidad de fracturas promedio similar de aproximadamente 17 fracturas/ metro. Este comportamiento en la intensidad de fracturas, ante una cantidad de deformación por extensión distinta, se atribuye al tipo de roca. La cantidad de extensión asociada a las dos familias de fracturas se distribuye de manera heterogénea en las rocas, lo que contribuye de manera significativa con la porosidad secundaria (porosidad de fracturas). Adicionalmente, la cantidad y el arreglo de las fracturas sugieren que las rocas miocénicas pueden ser la capa permeable del sistema geotérmico de la zona del lago de Cuitzeo.

Palabras clave: deformación frágil; fallas; fracturas; arreglo de fracturas; fractal; México.

\section{ABSTRACT}

Brittle deformation is accommodated by faults and fractures, which affect the upper crust of the Earth. The distribution of brittle deformation and the arrangement of faults and fractures are little known variables, although they influence the rock permeability and are important to understand the strain concentration. Our work explores the strain distribution and the fault arrangement on Miocene andesitic rocks in the Morelia-Cuitzeo area through a quantitative strain analysis at the mesoscopic scale. Two fracture families (F1 and F2) affect the rocks in the study area, and they are related to two brittle deformation events (D1 and D2) occurred between the Miocene and the Recent. The F1 fractures have an NNW-SSE strike and accommodate $2.5 \%$ of a ENE-WSW extension. The F2 fractures cut the F1 fractures, have an ENE-WSW strike, and accommodate $\sim 7 \%$ of a N-S extension. Both facture families have an arrangement of fracture clusters and average fracture intensity of 17 fractures/meter. The fracture intensity behavior is related to the rock type and not to the amount of strain. The finite strain (extension) of the F1 and F2 fractures has a heterogeneous distribution. This behavior contributes significantly to secondary porosity associated with F1 and F2 fractures. The strain distribution, and the quantity and arrangement of the fractures suggest that the Miocene rocks may be the permeable package of a geothermal system in the Cuitzeo Lake area.

Key words: fragile deformation; faults; fractures; arrangement of fractures; fractal; Mexico.

\section{INTRODUCCION}

La corteza superior de la Tierra suele deformarse en condiciones frágiles (Ramsay, 1967; Fossen, 2010). Esta deformación se manifiesta en las rocas a través de estructuras como fallas y fracturas en varias escalas (Cladouhos y Marrett, 1996; Vásquez-Serrano et al., 2019). La deformación frágil suele estar asociada a procesos de extensión, acortamiento y fenómenos como transpresión o transtensión (Ramsay, 1967; Fossen, 2010). En el caso particular del proceso de extensión en la corteza, las estructuras que se desarrollan son generalmente fallas normales y fracturas (extensionales, de cizalla e hibridas) (Ramsay, 1967; Ramsey y Chester, 2004; Vásquez-Serrano et al., 2019). La deformación frágil puede ser penetrativa en varias escalas dependiendo de la distribución y la intensidad de la deformación en las rocas (Ramsay, 1967; Cladouhos y Marrett, 1996; Fossen, 2010, Wang et al., 2019). La distribución de la deformación es una variable poco conocida, a pesar de que es fundamental para conocer la manera en cómo se acomoda la 
deformación (grado de heterogeneidad) y la relación que puede tener con la porosidad y permeabilidad en las rocas a través de la cantidad y arreglo que tienen las fracturas (Putz-Perrier y Sanderson, 2008).

En el área ubicada entre el lago de Cuitzeo y la ciudad de Morelia, Michoacán afloran rocas volcánicas del Mioceno afectadas por al menos dos eventos de deformación frágil. Dichos eventos generan dos familias de fallas con orientación NNW-SSE y ENE-WSW. La excelente exposición de estructuras frágiles permite explorar detalladamente la cantidad y el arreglo espacial que tienen las fracturas para conocer el potencial geotérmico de las rocas miocénicas en el área del lago de Cuitzeo, donde hay manifestaciones geotérmicas importantes (Bermejo-Santoyo, 2018).

Mediante un análisis cuantitativo de las fracturas dentro de la secuencia Tarímbaro, se analiza la manera en cómo se acomoda la deformación a través del método propuesto por Putz-Perrier y Sanderson (2008). Así mismo, se estima la cantidad, el arreglo y distribución espacial de las fracturas (Vásquez-Serrano; 2013; Vásquez-Serrano et al., 2019).

\section{MARCO GEOLÓGICO}

El área de estudio pertenece al flanco sur de la cuenca de Cuitzeo (Figura 1), la cual es una depresión tectónica que tiene una orientación ENE-WSW (Figura 1; Garduño-Monroy et al. 2009; Pola et al., 2016; Trujillo-Hernández, 2017; Bermejo-Santoyo, 2018; GómezVasconcelos et al., 2020). Se encuentra dentro del sector central de la Faja Volcánica Transmexicana (FVTM), un arco volcánico continental que se extiende por $\sim 1000 \mathrm{~km}$ de $\mathrm{W}$ a $\mathrm{E}$ en el centro de México y tiene una edad del Mioceno al Holoceno (García-Palomo et al., 2000; Gómez-Tuena y Carrasco-Núñez, 2000; Ferrari et al., 2011). La FVTM está relacionada a un proceso de subducción entre las placas de Cocos y Rivera con la placa Norteamericana (Figura 1a). Adicionalmente, su oblicuidad con respecto a la trinchera es una característica particular que la distingue de otros arcos volcánicos, la cual se asocia con variaciones en la velocidad y ángulo de subducción (Pardo y Suarez 1993, 1995; Ferrari et al., 2011; Manea et al., 2013).

En la región de Morelia-Cuitzeo, estudios detallados de la estra-
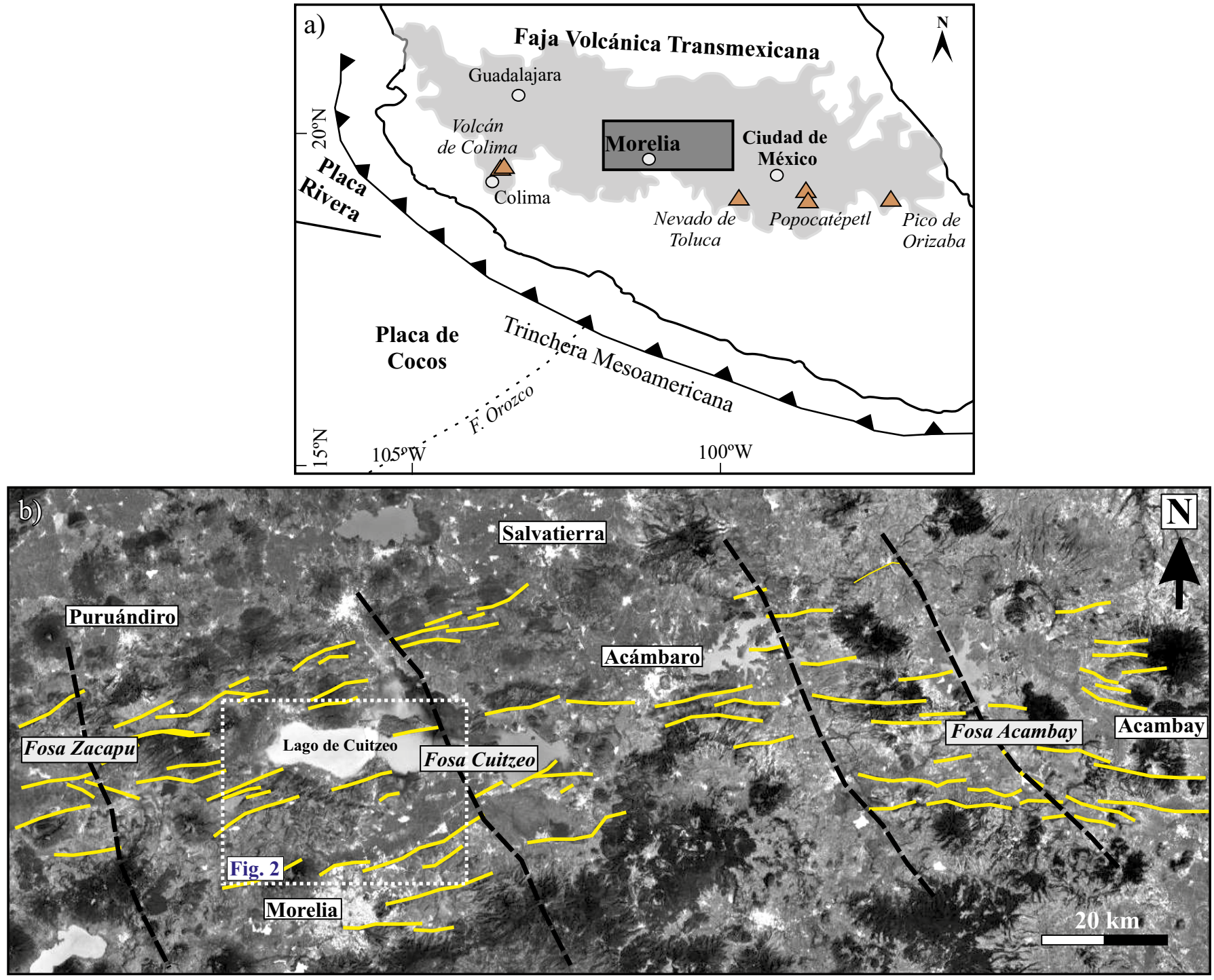

Figura 1. a) Mapa tectónico del centro-sur de México donde se muestra a la Faja Volcánica Transmexicana y la ubicación del área de estudio (Modificado de Arce et al., 2013). b) Mapa del Sistema de Fallas Morelia-Acambay ( E-W) y del sistema fallas NNW-SSW que afectan a los sectores este y central de la Faja Volcánica Transmexicana. Asimismo, se muestra la región Morelia-Cuitzeo donde se realizó nuestro trabajo (Modificado de Garduño-Monroy et al., 2009). 
tigrafía y de las estructuras que afectan a las sucesiones volcánicas del Mioceno al Holoceno (Garduño-Monroy et al., 2009; TrujilloHernández, 2017; Bermejo-Santoyo, 2018; Mazzoldi et al., 2020) indican el registro de dos eventos de actividad tectónica extensional relacionada con las abundantes fracturas que se observan en dicha región (Garduño-Monroy et al., 2009).

\section{Estratigrafía}

La unidad más antigua dentro del área de Morelia-Cuitzeo es una sucesión de rocas siliciclásticas del Jurásico Medio-Cretácico (Pasquarè et al., 1991). Sobreyaciendo discordantemente a esta unidad se encuentran conglomerados, areniscas y depósitos fluviales del Grupo Balsas del Paleoceno (Pasquarè et al., 1991; Garduño-Monroy et al., 2009). Por arriba del Grupo Balsas, se encuentra una sucesión de rocas volcánicas y depósitos volcaniclásticos que se extienden hasta el Oligoceno-Mioceno. Toda esta sucesión no aflora en el área de estudio, pero está expuesta hacia el SE, en la región de Tzitzio, Michoacán (Pasquarè et al., 1991). Cubriendo a esta última sucesión, existen una serie de secuencias volcánicas relacionadas a la actividad volcánica de la Sierra Madre Occidental y Faja Volcánica Transmexicana (Figura 2) (Garduño-Monroy et al., 2009; Gómez-Vasconcelos et al., 2015; Avellán et al., 2020; Gómez-Vasconcelos et al., 2020). La secuencia más antigua la constituyen rocas de la Sierra Mil Cumbres, la cual está representada por una sucesión de lavas andesíticas y dacíticas, además de depósitos piroclásticos del Mioceno Temprano (Silva-Mora, 1995; Garduño-Monroy et al., 2009; Gómez-Vasconcelos et al., 2015; Trujillo-Hernández, 2017; Avellán et al., 2020; Gómez-Vasconcelos et al., 2020). La sucesión continúa con una secuencia de ignimbritas llamada Copándaro, la cual aflora al oeste del área de estudio (Pola et al., 2016; Trujillo-Hernández, 2017). Estas ignimbritas son sobreyacidas por la secuencia Tarímbaro, la cual está constituida por lavas andesíticas y traquiandesíticas del Mioceno Temprano (Trujillo-Hernández, 2017). Esta secuencia está ampliamente distribuida dentro del área de estudio (Figura 2) y es la que se usa para el análisis cuantitativo de la deformación en el presente trabajo. La secuencia Tarímbaro equivale en edad a las lavas Cuitzeo reportadas por Avellán et al. (2020). Descansan sobre la secuencia Tarímbaro, las secuencias Chucándiro y Santa Rita, conformadas por ignimbritas del Mioceno Temprano y lavas andesíticas del Mioceno Medio-Tardío, respectivamente (TrujilloHernández, 2017).

La sucesión volcánica continúa con la secuencia monogenética del Mioceno Tardío-Pleistoceno, representada por volcanes de composición andesítico-basáltica (Figura 2. Garduño-Monroy et al., 2009; Gómez-Vasconcelos et al., 2015; Trujillo-Hernández, 2017). Finalmente, sobreyaciendo a las sucesiones volcánicas existen depósitos volcaniclásticos y lacustres cuaternarios. Estos últimos asociados con el desarrollo del lago de Cuitzeo (Figura 2. Trujillo-Hernández, 2017).

\section{Estructuras mayores}

Dentro de la región de Morelia-Cuitzeo, las rocas volcánicas del Mioceno están afectadas por dos sistemas de fallas principales. El sistema más antiguo tiene una orientación preferencial NNW-SSE con una cinemática de falla lateral derecha con componente normal (Suter et al., 1995a, 1995b; 1996; Mennella et al., 2000; Alaniz-Álvarez et al., 2002b; Alaniz-Álvarez y Nieto-Samaniego, 2005 Garduño-Monroy et al., 2009; Bermejo-Santoyo, 2018; Gómez-Vasconcelos et al., 2021). Una de las estructuras más representativas de este sistema, es la falla Cuto del Porvenir, la cual se ubica en el centro del área de estudio (Figura 2). Esta falla corta a la secuencia Santa Rita, lo cual sugiere una actividad restringida al Mioceno-Plioceno (Figura 2). El sistema de fallas NNW-

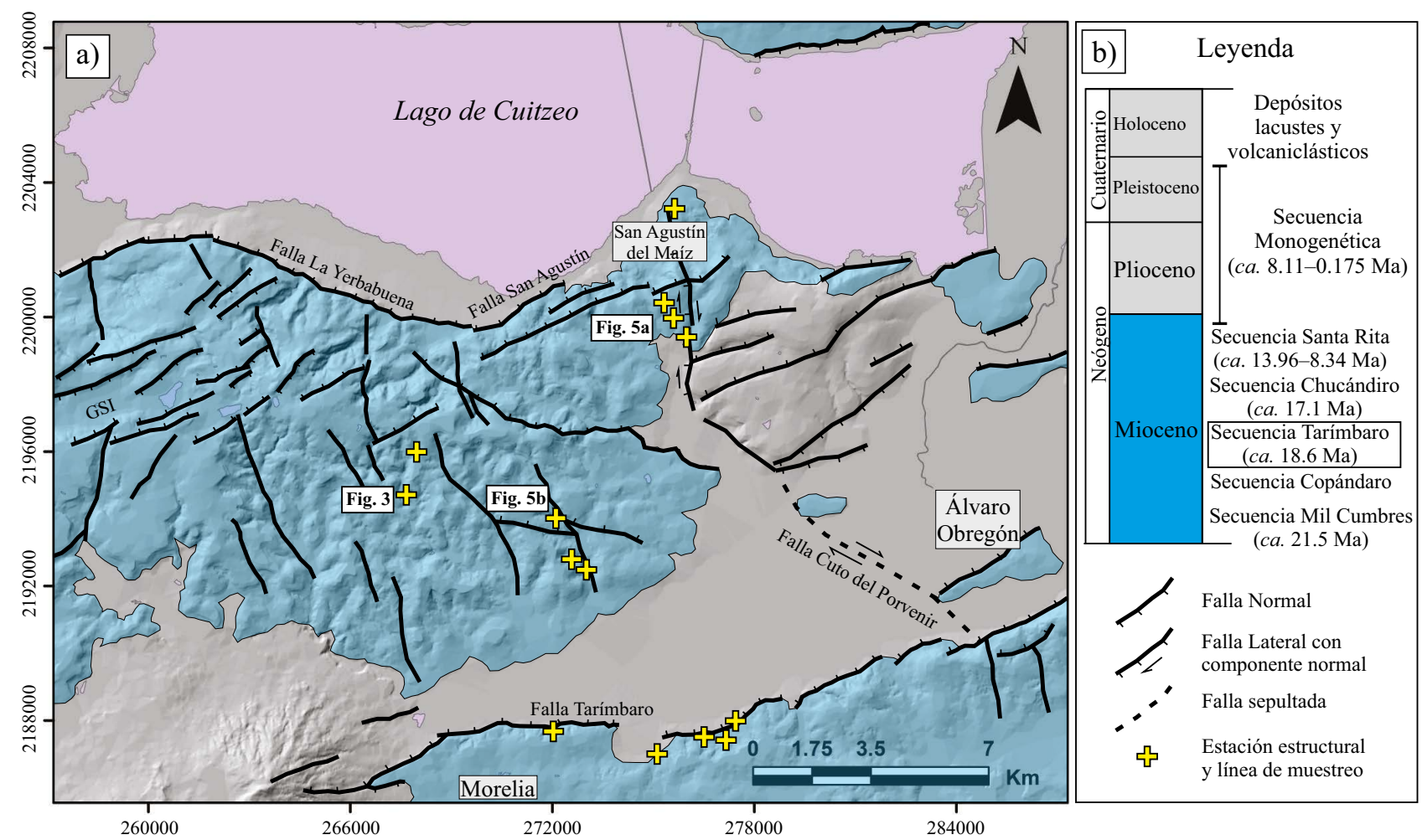

Figura 2. a) Mapa del área de Morelia-Cuitzeo donde se muestra la distribución de las rocas miocénicas y los principales sistemas de fallas que las afectan (Modificado de Trujillo-Hernández, 2017Trujillo-Hernández, 2017b) Columna estratigráfica general del área de estudio basada en edades $\mathrm{Ar}^{40}$ - $\mathrm{Ar}^{39}$ del trabajo de (TrujilloHernández, 2017). Adicionalmente, se ubican los puntos donde se realizaron las líneas de muestreo y levantamiento de datos estructurales. 
SSE, se ha interpretado que pertenece al evento tectónico que formó a la provincia de Sierras y Cuencas en la porción noroeste de México (Suter et al., 1996; Mennella et al., 2000; Alaniz-Álvarez y Nieto-Samaniego, 2005; Garduño-Monroy et al., 2009). Esta provincia es el resultado de la extensión cortical ocurrida durante el Oligoceno-Mioceno (Henry y Aranda-Gómez., 1992; Garduño-Monroy et al., 2009). Al sur del estado de Guanajuato, dentro del área de estudio, la actividad del sistema NNW-SSE se ha interpretado que sucedió durante el Mioceno Medio-Tardío debido a que las fallas cortan a una secuencia basáltica del Mioceno, la cual es sobreyacida por vulcanismo riolítico de entre 6.1 y $2.8 \mathrm{Ma}$ (fechado por el método de K-Ar) que no se encuentra afectado por dichas fallas (Pasquarè et al., 1991).

El sistema de fallas más joven está representado por estructuras que tienen un rumbo preferencial ENE-WSW con cinemática principalmente de tipo normal (Suter et al., 1995a, 1995b; 1996; GarduñoMonroy et al., 2009). Estas estructuras pertenecen al Sistema de Fallas Morelia-Acambay (SFMA), el cual afecta al sector central y oriental de la FVTM y forma un conjunto de fosas tectónicas (por ejemplo, las Fosas de Acambay y Cuitzeo) que son ocupadas por lagos (Figuras 1 y 2) (Martínez-Reyes y Nieto-Samaniego, 1990; Suter et al., 1995a, 1995b, 1996; Ferrari 2000). El SFMA se considera activo debido a la actividad sísmica reciente en la región de Acambay (Urbina y Camacho, 1913) y Maravatío (Astíz-Delgado, 1980; Rodríguez-Pascua et al., 2017), y a los trabajos de paleosismología y tectónica realizados en los últimos años (Langridge et al., 2000; 2013; Garduño-Monroy et al., 2009; SunyePuchol et al., 2015; Ortuño et al., 2015; 2018; Suter, 2015; Lacan et al., 2018, Soria-Caballero et al., 2019), aunque su actividad probablemente inició desde el Mioceno Tardío (Garduño-Monroy et al., 2009). En la región de Morelia-Cuitzeo, las estructuras del SFMA cortan a las fallas NNW-SSW y basculan hacia el sur a la sucesión volcánica del Mioceno (Garduño-Monroy et al., 2009; Mazzoldi et al., 2020).

\section{METODOLOGÍA}

Nuestro trabajo se enfocó en el estudio de las estructuras frágiles que afectan a las rocas andesíticas de la secuencia Tarímbaro, debido a que son un análogo expuesto de las rocas que representan el yacimiento geotérmico del área del lago Cuitzeo (Bermejo-Santoyo, 2018), donde tienen un espesor de aproximadamente $2 \mathrm{~km}$ (Mazzoldi et al., 2020).

A continuación, se expone la metodología del levantamiento de datos en campo de variables como apertura y el espaciamiento entre fracturas. Asimismo, se describen los métodos para el análisis de la distribución y arreglo de fracturas, y la estimación de la deformación.

\section{Levantamiento de datos en el campo}

El análisis cuantitativo de la deformación puede realizarse en una dimensión mediante el uso de líneas de muestreo (Figura 3. PutzPerrier y Sanderson, 2008). Estas líneas de muestreo son trazadas en los afloramientos de manera perpendicular al rumbo de una familia de fracturas (conjunto de fracturas paralelas) (La Pointe y Hudson, 1985). Este procedimiento asegura que los datos de espaciamiento entre fracturas y apertura sean medidos adecuadamente (Vásquez-Serrano et al., 2019). Para nuestro estudio, se hizo un levantamiento sistemático de líneas de muestreo en rocas andesíticas de la secuencia Tarímbaro dentro del área comprendida entre la ciudad de Morelia y el lago de Cuitzeo (Figura 2). Se midió un total de 24 líneas de muestreo con una longitud de entre $0.9 \mathrm{y} \sim 2 \mathrm{~m}, 14$ de ellas en las fracturas asociadas con las fallas NNW-SSE y 10 en fracturas relacionadas con las fallas ENE-WSW. La elección de afloramientos para la realización de las líneas de muestreo tomó en cuenta las recomendaciones propuestas por Vásquez-Serrano (2013), asociadas con la cantidad y el arreglo de las fracturas geológicas.

Tanto la apertura (distancia entre paredes de una fractura) como el espaciamiento (distancia entre fracturas) fueron medidos simultáneamente en cada línea de muestreo como se muestra en la Figura 3. Este procedimiento nos permitió obtener una lista de aperturas y espaciamientos para cada línea de muestreo, lo cual es fundamental para conocer la distribución de las fracturas y la deformación que acomodan (extensión perpendicular a las paredes de las fracturas) (Putz-Perrier y Sanderson, 2008; Vásquez-Serrano et al., 2019). En el levantamiento de los datos, las líneas de muestreo incluyeron fracturas extensionales e híbridas (extensión y cizalla) (Fossen, 2010) asociadas con los dos sistemas de fallas observadas dentro del área de estudio. Es

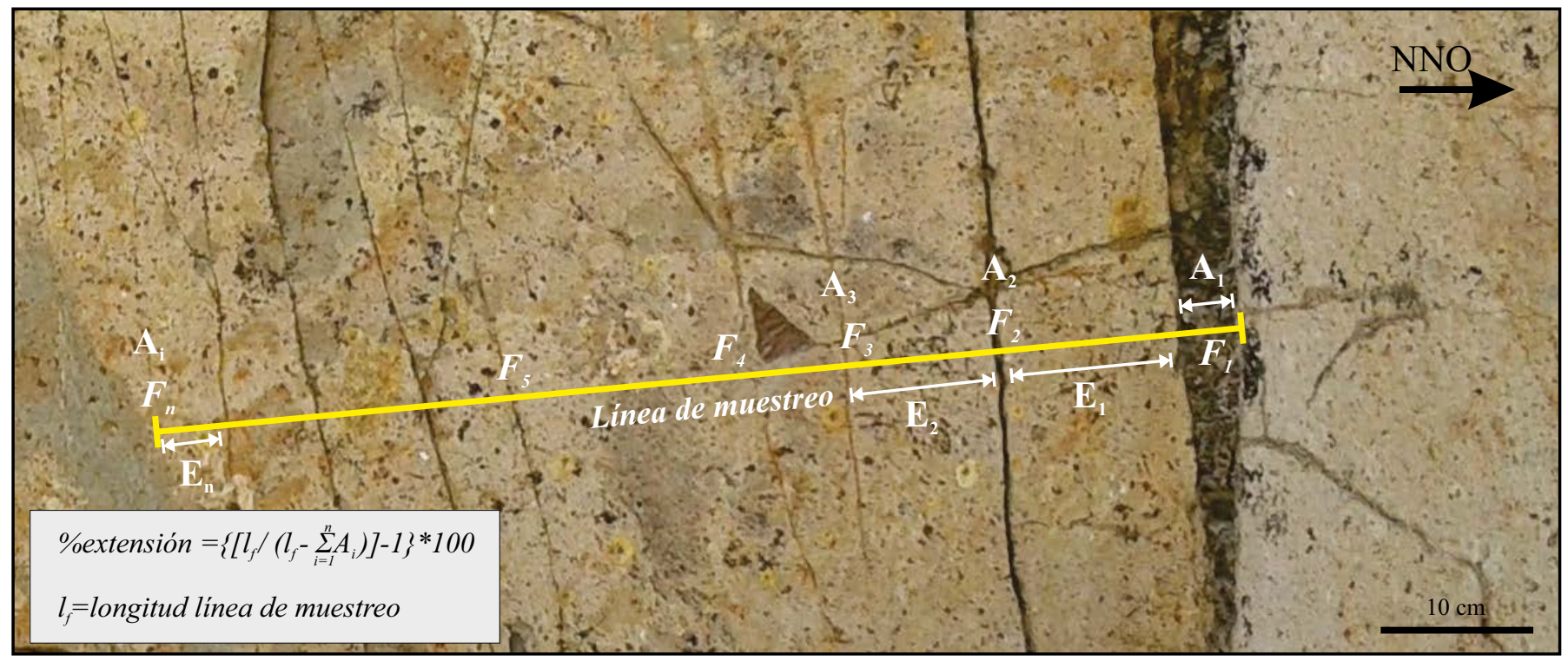

Figura 3. Método de obtención de los datos de espaciamiento (distancia entre fracturas) y apertura (distancia entre paredes de una fractura) a través de una línea de muestreo, y ecuación para la estimación de la extensión finita usando los datos de apertura. Afloramiento de fracturas ENE-WSW en la región Morelia-Cuitzeo. 
importante mencionar que no se contabilizó el desplazamiento paralelo a las paredes de las fracturas híbridas.

Adicionalmente, se midió la orientación e inclinación de las fracturas, con el fin de conocer las diferentes familias de fracturas y compararlas con los sistemas de fallas reportados en la región Morelia-Cuitzeo. Finalmente, se realizó una descripción detallada de la geometría de las fracturas observadas en cada sitio (máxima apertura, longitud, relaciones de corte). Esto último debido a que las fracturas asociadas a los dos eventos de deformación frágil presentan características distintas. Un aspecto importante, es que nuestro estudio no considera a las fracturas laminadas (relacionadas con el flujo y enfriamiento de flujos de lava) observadas en las rocas andesíticas de la secuencia Tarímbaro, solo a las fracturas asociadas a los dos sistemas de fallas reportados para la zona de estudio.

\section{Análisis fractal}

Las fracturas geológicas cumplen con una característica esencial de los objetos fractales: la invariancia en la escala, es decir, que todas sus características geométricas (longitud, apertura, orientación) se repiten en varias escalas (Mandelbrot, 1967). Asimismo, los objetos fractales se caracterizan por presentar una función de ley de potencia en la relación cantidad-tamaño (Barton y LaPointe, 1995; Cladouhos y Marrett, 1996; Marrett et al., 1999). Se ha demostrado que el espaciamiento entre las fracturas y la apertura, son variables que pueden ser analizadas a través de las técnicas de la teoría fractal, ya que siguen una ley de potencia en la relación cantidad-tamaño (Hooker et al., 2013, 2018; Laubach et al., 2018; Vásquez-Serrano et al., 2019). Esto último se puede demostrar usando gráficos bilogarítmicos del espaciamiento y apertura contra su frecuencia acumulada (Barton y LaPointe, 1995).

En este trabajo, se utilizan herramientas de la teoría fractal para analizar el arreglo y la distribución que tienen las fracturas geológicas. Los parámetros fractales que se usan para dicho propósito son: dimensión de caja, correlación y exponente de Lyapunov (Lv). La dimensión de caja y correlación están relacionadas con la cantidad de fracturas y con la manera en cómo éstas ocupan el espacio que las contienen (Barton y LaPointe, 1995; Riley et al., 2011; Vásquez-Serrano et al., 2019). Por otro lado, el exponente de Lyapunov depende del grado de agrupamiento de las fracturas, $s i \mathrm{Lv}=0$ se tendrán espaciamientos iguales entre las fracturas, mientras que si $\mathrm{Lv}>0$ existirán grupos de fracturas (Riley et al., 2011; Vásquez-Serrano et al., 2019). Adicionalmente, se calcula la intensidad de fracturas (número de fracturas por unidad de longitud) y el coeficiente de variación (Cv). Este último parámetro se obtiene del cociente de la desviación estándar con el promedio de los espaciamientos; si el valor $\mathrm{de} \mathrm{Cv}=0$ la distancia entre fracturas es igual, y cuando $\mathrm{Cv}>0$ las fracturas tendrán una distribución aleatoria $(\mathrm{Cv}=1)$ o se agruparán $(\mathrm{Cv}>1)$ (Vásquez-Serrano, 2013). Adicionalmente, se estimó el parámetro $\mathrm{Cv}^{*}$, el cual es usado generalmente para un número de datos de espaciamiento $(n)$ reducido. Este parámetro esta dado por la ecuación: $\mathrm{Cv}^{*}=\mathrm{Cv}[(\mathrm{n}+1) /(\mathrm{n}-1)]^{1 / 2}$ y es aplicado para caracterizar la evolución espacial de fracturas y fallas (Gillespie et al., 2001).

Todos estos parámetros fueron calculados siguiendo la metodología descrita por Vásquez-Serrano (2013) y Vásquez-Serrano et al. (2019) tomando como base las teorías propuestas por Cox y Lewis (1966), Davy et al. (1990), Rosenstein et al. (1993), Barton y LaPointe (1995) y Riley et al. (2011). El valor de dichos parámetros se obtuvo a través del código computacional Frackita escrito en MATLAB por Vásquez-Serrano (2020).

\section{Estimación de la deformación finita}

El conocimiento de la distribución de las estructuras frágiles (fallas y fracturas) en conjunto con la cantidad de deformación que acomodan, es fundamental para analizar fenómenos como el flujo de fluidos, la evolución de un grupo de fracturas y la deformación localizada en la corteza (Evans et al., 1997; Billi et al., 2003).

En este trabajo se hace una estimación de la deformación finita y un análisis de su distribución para los dos eventos de deformación frágil. Para el caso de la estimación de la deformación (extensión) se usan los datos de apertura de cada línea de muestreo (Figura 3) a través de la siguiente ecuación (Ramsay, 1967):

$$
\text { \%extension }=\left\{\left[\frac{l f}{l f-\sum_{i=1}^{n} A i}\right]-1\right\} \times 100
$$

Aquí $l_{f}$ es la longitud de la línea de muestreo y $A_{1}, A_{2}, A_{3} \ldots A_{i}$ corresponde la apertura de cada una de las fracturas cortadas por la línea de muestreo (Figura 3).

Si se analiza la distribución de dos familias de fracturas en tres escalas distintas con diferentes cantidades de extensión (Figuras 4a y $4 \mathrm{~b})$, es posible determinar el grado de heterogeneidad en la distribución de la deformación usando gráficos de apertura acumulada contra la distancia (Figura 4c), y a través del parámetro $V s^{\prime}$ y su relación con la apertura máxima (Figura $4 \mathrm{~d}$ ). El valor de $V s^{\prime}$ se obtiene a partir de gráficos de apertura acumulada contra la distancia, donde se determina la desviación máxima positiva y negativa $\left(D^{+}\right.$y $\left.D^{-}\right)$de la función obtenida a partir de los datos de apertura, comparándola con la función de una distribución homogénea en la deformación (Figura 4e). Vs' se obtiene a partir de la siguiente ecuación (Putz-Perrier y Sanderson, 2008):

$$
V s^{\prime}=\frac{V}{T}
$$

En la ecuación anterior, T es el valor máximo de la apertura acumulada (Figura 4) y V se obtiene a partir de la prueba de Kuiper (1960), donde se compara la distribución de un grupo de datos observados con una distribución homogénea (Figura 4e). Kuiper (1960) sugiere que $V=\left|D^{+}\right|+\left|D^{-}\right|$en un gráfico de datos acumulados (Figura 4e). $D^{+}$ y $D^{-}$se puede estimar con el gráfico de apertura acumulada contra la distancia (Putz-Perrier y Sanderson, 2008). Si Vs' se aproxima a cero se tendrá una distribución homogénea en la deformación frágil, en caso contrario, si $V s^{\prime}$ tiene valores mayores a cero la deformación se distribuirá de manera heterogénea (Figura 4d). Es importante señalar que con base en los ejemplos de la Figura 4, el valor del parámetro $V s^{\prime}$ también puede depender de la escala de observación (Putz-Perrier y Sanderson, 2008), por eso es importante especificar la escala de análisis de la deformación en las estructuras frágiles (fracturas y fallas).

\section{RESULTADOS}

\section{Características de las fracturas}

Las fracturas que afectan a las rocas andesíticas de la secuencia Tarímbaro son principalmente híbridas y extensionales. Las fracturas híbridas son las más numerosas en las rocas andesíticas. Mientras que las fracturas extensionales son las que acomodan la mayor deformación por extensión y se encuentran rellenas de material arcilloso de color café-verdoso (Figura 3). Además de las fracturas ligadas a las fallas (híbridas y extensionales), también hay una presencia importante de fracturas laminadas subhorizontales (Magnall, 2018). Estas fracturas están cortadas, basculadas y reactivadas por la fracturas híbridas y extensionales (Figura 5).

La orientación de las fracturas híbridas y extensionales subverticales, así como sus relaciones de corte permiten agrupar a las fracturas en dos familias (Figura 5). Por un lado, está la familia F1 que tiene un rumbo preferencial NNW-SSE, corta a las fracturas laminadas y es cortada por la familia F2 (Figura 5). La familia F2 tiene un rumbo 
a)

$\mathrm{a}_{3}$ ) extensión $=10.65 \%$

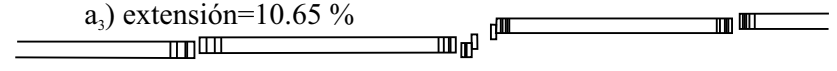

$a_{3}$ ) extensión $=4.65 \%$

$\mathrm{a}_{3}$ ) extensión $=1.05 \%$

b)

$b_{1}$ ) extensión $=10.65 \%$

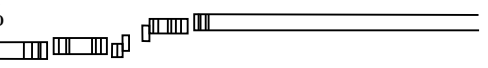
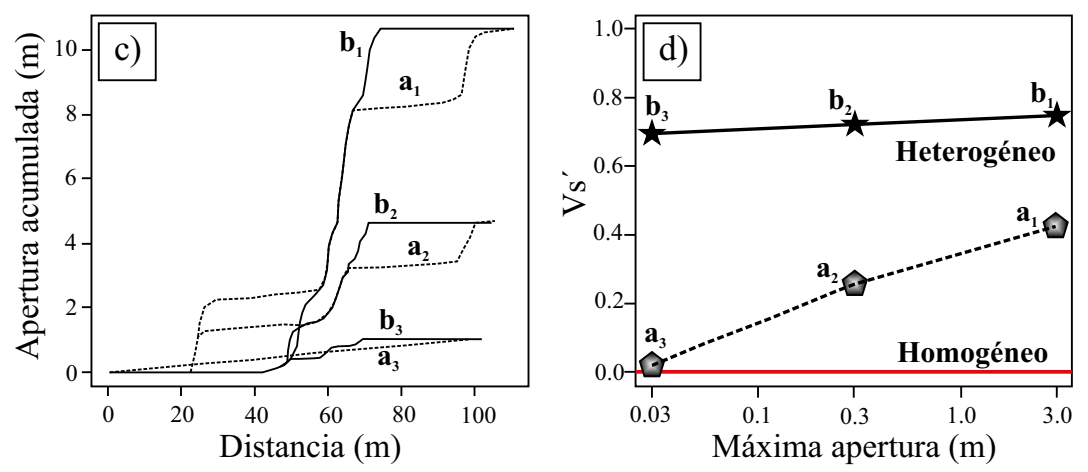

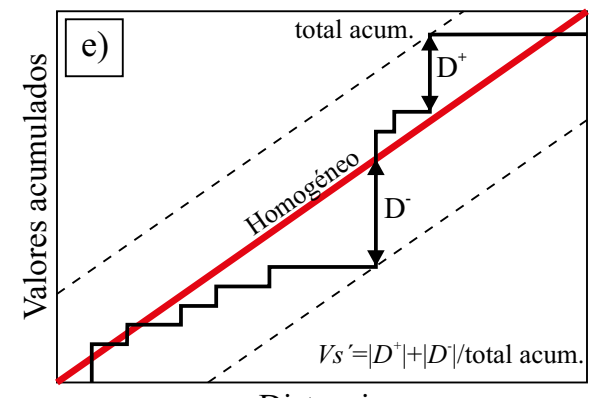

Distancia

Figura 4. a) y b) Ejemplos de fracturas sintéticas a distintas escalas con diferentes cantidades de deformación y modos de distribución de dicha deformación (tomado de Putz-Perrier y Sanderson, 2008). c) Gráfico de apertura acumulada de la apertura contra la distancia para cada uno de los ejemplos de fracturas sintéticas. Obsérvese que el valor máximo de apertura acumulada está asociado con la cantidad de deformación que genera a cada conjunto de fracturas. d) Gráfico del parámetro $V s^{\prime}$ (grado de heterogeneidad en la distribución de la deformación) contra la apertura máxima, donde se puede diferenciar entre distribuciones de deformación homogénea y heterogénea. e) Método de estimación del parámetro $V s^{\prime}$ mediante gráficos de apertura acumulada. En este grafico la línea roca representa la distribución uniforme de la deformación y la línea negra la distribución de los datos de apertura (los gráficos son tomados de Putz-Perrier y Sanderson, 2008).

ENE-WSW y corta a las fracturas de la familia F1 y a las fracturas laminadas (Figura 5). Una característica distintiva de las fracturas F2, es que parte de la deformación que acomodan es repartida a las fracturas laminadas, las cuales se reactivan como fracturas de cizalla y generan estructuras en echelon (Figura 5) (Fossen, 2010). Estas estructuras son subhorizontales y tienen un rumbo paralelo a las fracturas F2.

\section{Parámetros fractales}

En general, las fracturas de las familias F1 y F2 muestran un arreglo de fracturas agrupadas en las líneas de muestreo medidas en cada afloramiento (Figura 6a). Nuestro análisis de los parámetros fractales sugiere que el exponente de Lyapunov tiene un promedio de 1.32 para las fracturas F1 y 1.46 para las fracturas F2 (Tablas 1 y 2). Por otro lado, el coeficiente de variación $(\mathrm{Cv})$ muestra valores promedio de 0.75 y 0.81 para las fracturas $\mathrm{F} 1$ y F2, respectivamente. Los valores del parámetro $\mathrm{Cv}^{*}$ para las fracturas F1 y F2 tienen valores promedio de 0.80 y 0.86 , respectivamente (Tablas 1 y 2 ). Para el caso de la dimensión fractal de caja, las fracturas F1 muestran un valor promedio 0.52 , mientras que las fracturas F2 tienen un valor promedio de 0.49 (Tablas 1 y 2). Así mismo, en la dimensión de correlación, se obtuvieron valores de 0.50 para las fracturas F1 y 0.48 para las fracturas F2 (Tablas 1 y 2).

La apertura y el espaciamiento entre fracturas son variables que se pueden analizar en un rango de escala dado, a través de un gráfico bilogarítmico de frecuencia acumulada contra el tamaño, donde la pendiente (exponente de frecuencia acumulada) mostrará la proporción cantidad-tamaño de estas variables. Tanto la apertura como el espaciamiento en las fracturas analizadas muestran una relación frecuencia-tamaño ligada a una función de potencia (Figura 6). En los gráficos de la Figura $6 \mathrm{~b}$ y $6 \mathrm{c}$, es posible observar que las fracturas F1 tienen valores del exponente de frecuencia acumulada de la apertura y del espaciamiento, más grandes que las fracturas F2. Para el caso específico de la apertura, los valores del exponente de frecuencia acumulada son de 2.216 y 1.761 para las fracturas F1 y F2, respectivamente. Adicionalmente, los valores de apertura máxima medidos en cada afloramiento, muestran que las fracturas F1 tiene una apertura promedio $(0.4 \mathrm{~cm})$, menor en comparación con las fracturas F2 (apertura máxima promedio $=1.8 \mathrm{~cm})($ Tablas 1 y 2). En lo que respecta al espaciamiento, el exponente de frecuencia acumulada para las fracturas F1 es de 2.06, mientras que para las fracturas F2 es de 1.259 (Figura 6). La intensidad de fracturas tiene valores promedio muy similares en las dos familias de fracturas, las fracturas F1 tienen una intensidad de 17.79 (fracturas/metro), mientras que las fracturas F2 tienen un valor promedio en la intensidad de 16.50 (fracturas/metro) (Tablas 1 y 2).

\section{Deformación asociada a las fracturas y su distribución}

Una parte importante del presente trabajo es el análisis cuantitativo de la deformación. Para lograr este propósito se estimó la extensión finita asociada con las fracturas de las familias F1 y F2 (Figura 3 y Tablas 1 y 2). Es preciso aclarar que no se midió el desplazamiento paralelo a las paredes (deformación por cizalla) de las fracturas híbridas ni la deformación repartida a las fracturas laminadas por parte de las fracturas F2 (Figura 5). En cuanto a la cantidad de extensión que 
acomodan las fracturas (medida en una dimensión), las fracturas F1 acomodan una menor deformación en comparación con las fracturas F2 a escala mesoscópica (Tablas 1 y 2). Las fracturas F1 acomodan una extensión ENE-WSW de alrededor de $2.46 \%$, mientras que las fracturas F2 tienen una extensión NNW-SSE promedio de $6.84 \%$. La extensión en las fracturas $\mathrm{F} 1$ es acomodada principalmente a través de fracturas híbridas, contrariamente a lo que sucede con las fracturas F2, donde la extensión se acomoda en las fracturas extensionales.

Los gráficos de apertura acumulada contra la distancia son muy similares para las dos familias de fracturas estudiadas (F1 y F2). En ambos casos, se observan variaciones de los datos medidos alrededor de la función que representanta una distribución homogénea de la deformación (Figura 7a y 7b). En las fracturas F1, el parámetro Vs' tiene un valor promedio de 0.23 . Por otro lado, las fracturas F2 presentan un valor promedio de $V s^{\prime}$ de 0.33 (Tablas 1 y 2 ).

Ambas familias de fracturas (F1 y F2) tienden a una distribución más homogénea en la deformación conforme aumenta el número de fracturas en las líneas de muestreo (7c), aunque conservan cierto grado de heterogeneidad (Figura 7d). Algunos valores de $V s^{\prime}$ para las fracturas
F2 muestran un grado de heterogeneidad alto en comparación con los valores $V s^{\prime}$ de las fracturas F1 (Figura 7c). Los valores altos de $V s^{\prime}$ en las fracturas F2 también están asociados con valores altos en la apertura máxima como se puede observar en la Figura $7 d$.

\section{DISCUSION}

\section{Distribución de fracturas y deformación frágil}

En el área de Morelia-Cuitzeo, las rocas andesíticas del Mioceno se encuentran afectadas por dos familias de fracturas (F1 y F2) a escala mesoscópica, asociadas con dos eventos de deformación frágil. $\mathrm{El}$ análisis de aperturas y espaciamientos entre fracturas, para las dos familias de fracturas estudiadas (F1 y F2), sugiere que las fracturas F1 están dominadas por aperturas inferiores a $0.7 \mathrm{~cm}$, mientras que las fracturas F2 presentan una cantidad considerable de aperturas entre 1 y $2 \mathrm{~cm}$ (Figuras $6 \mathrm{~b}$ y 8 ). En el caso del espaciamiento, el gráfico de frecuencia acumulada muestra que las fracturas $\mathrm{F} 1$ presentan una cantidad mayor de espaciamientos pequeños. Contrariamente, las fracturas F2
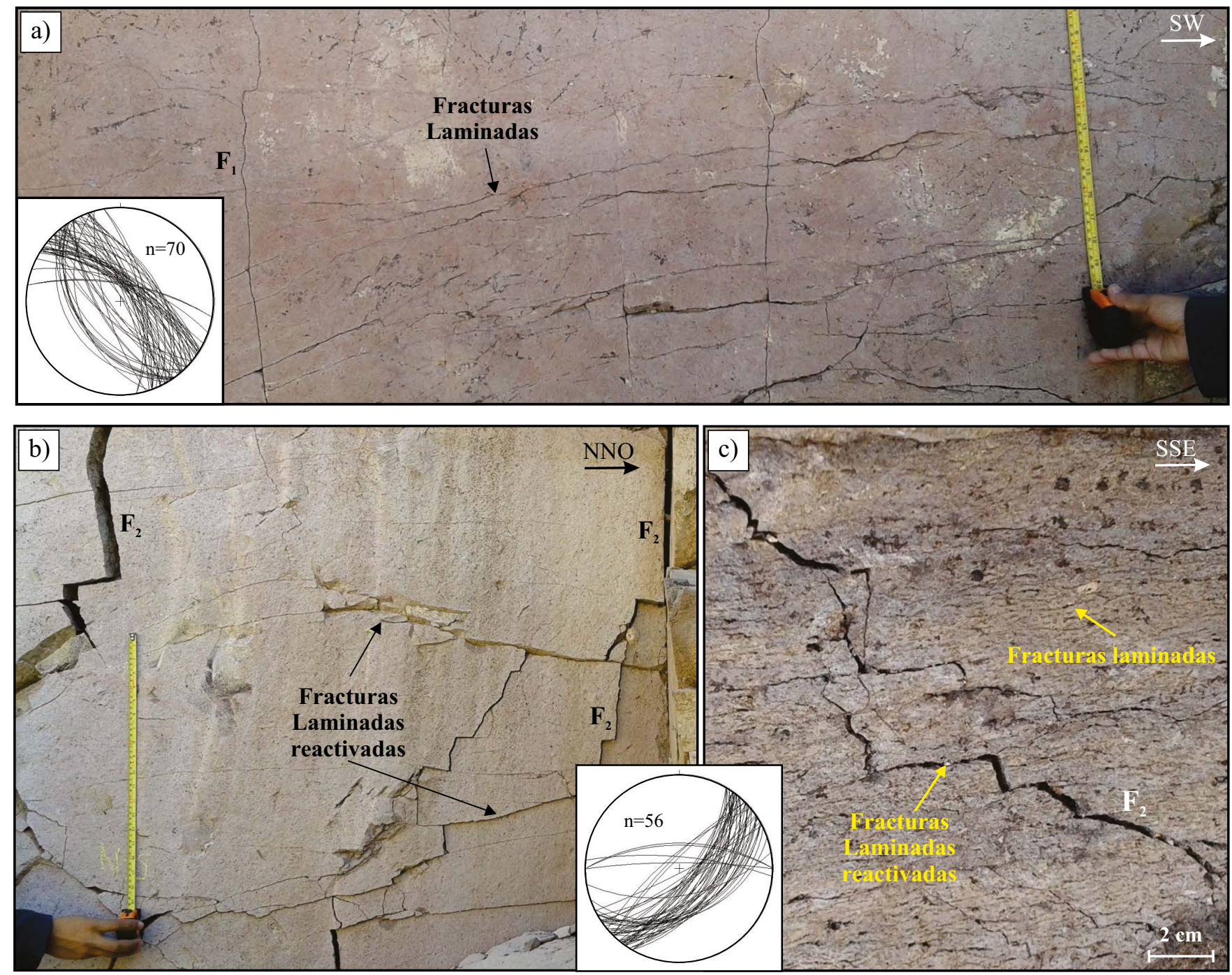

Figura 5. a) y b) Características principales de las familias de fracturas F1 y F2 que afectan a las rocas andesíticas de la secuencia Tarímbaro, y su orientación dentro del área de estudio. c) Asimismo, es posible observar a las fracturas laminadas y su relación con las fracturas F1 y F2. Nótese que las fracturas laminadas son reactivadas en conjunto con la formación de las fracturas F2. Asimismo, se presentan dos redes estereográficas donde se grafican los planos de fracturas de las familias F1 y F2. 
tienden a estar separadas por espaciamientos más grandes (mayores a $20 \mathrm{~cm}$ ) (Figura $6 \mathrm{c}$ y 8 ). Los valores de exponente de frecuencia acumulada de la apertura tienen valores similares a los reportados en otros sitios dentro del Cinturón Volcánico Trans-Mexicano (cuenca de México) para rocas andesíticas del Mioceno (Vásquez-Serrano et al., 2019 y Vásquez-Serrano, 2020). Además, es interesante que las fracturas NNW-SSE presenten los valores más altos en el exponente de frecuencia acumulada del espaciamiento en comparación con las fracturas ENE-WSW para el mismo tipo de roca y de la misma edad en la cuenca de México (Vásquez-Serrano et al., 2019; Vásquez-Serrano, 2020). El comportamiento de la apertura y el espaciamiento, sugieren que estas variables están ligadas más a la deformación relacionada a un evento tectónico que al tipo de roca que afecta dicho evento.

Los gráficos de distribución de fracturas muestran que ambas familias de fracturas (F1 y F2) tienen un arreglo agrupado (Figura 6a). Esto es confirmado con los valores obtenidos del exponente de Lyapunov en cada una de las líneas de muestreo (Tablas 1 y 2). Los valores prome- dio del exponente de Lyapunov, sugieren que las fracturas F2 tienden a formar grupos de fracturas más definidos en comparación con las fracturas F1. El arreglo agrupado de las fracturas F1 y F2 también es corroborado con los valores del coeficiente de correlación (Tablas 1 y 2, y Figura 8). Nuestro análisis sugiere que la dimensión fractal de caja y correlación tienen valores similares para ambas familias de fracturas (Tablas 1 y 2). Este comportamiento también ha sido observado en rocas calcáreas (Vásquez-Serrano, 2013), y se relaciona con la distribución (arreglo) y cantidad de fracturas (Barton y LaPointe, 1995; VásquezSerrano, 2013). En ambas familias de fracturas (F1 y F2), se tiene una intensidad de fracturas muy similar, lo que puede estar asociado con la cantidad máxima de fracturas que puede contener un tipo de roca en específico, ante un evento de deformación. La existencia de un límite máximo en la cantidad de fracturas también ha sido reportado en otros trabajos para rocas sedimentaria (caliza) e ígneas intrusivas (granito) (Nieto-Samaniego et al., 2003, 2005; Vásquez-Serrano, 2013). Los resultados obtenidos en los parámetros: dimensión de caja, dimensión

a)

Fracturas F1

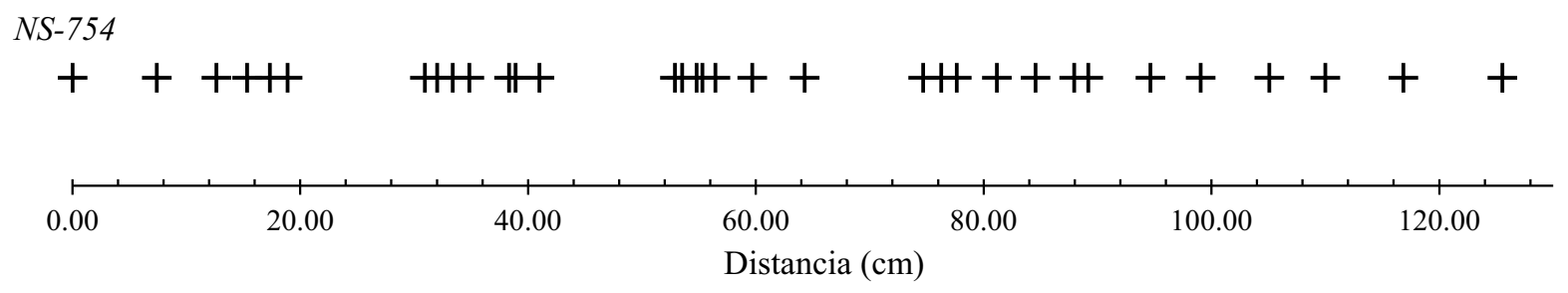

Fracturas F2

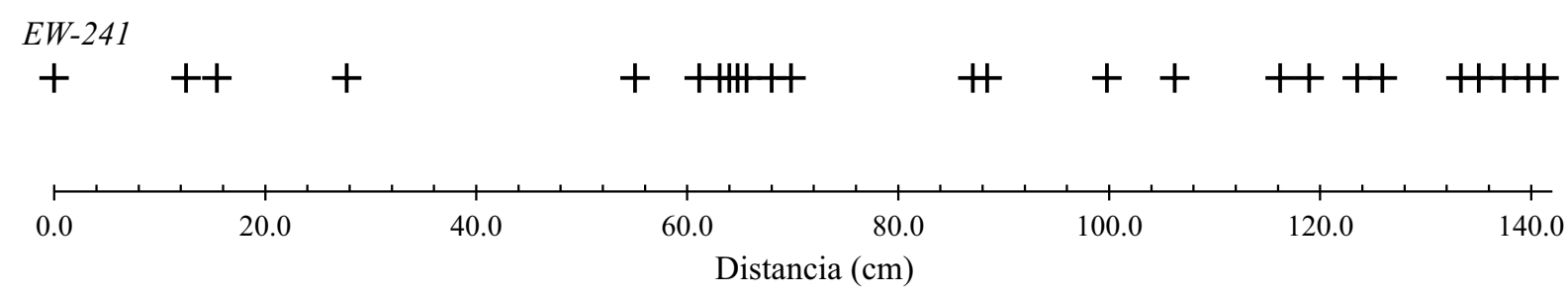

b)

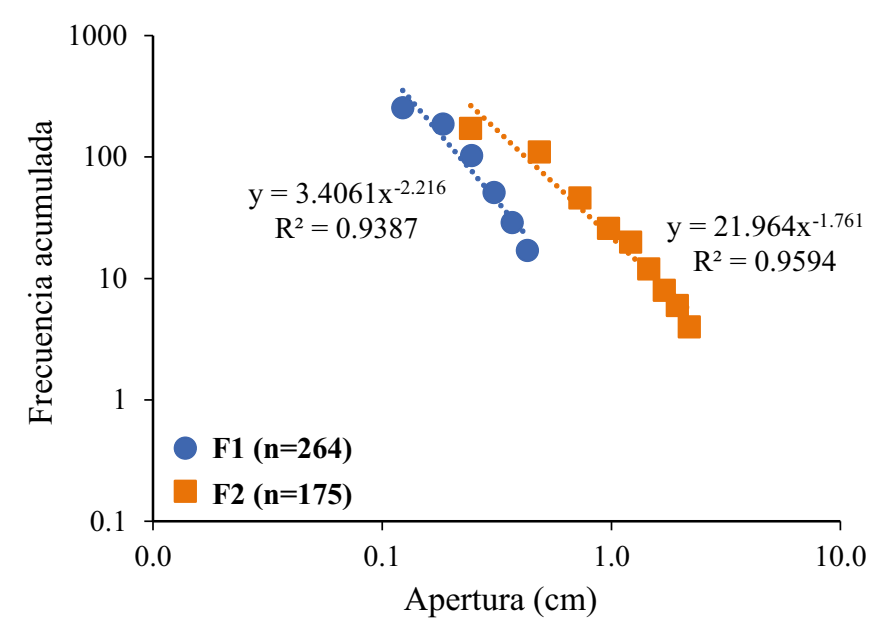

c)

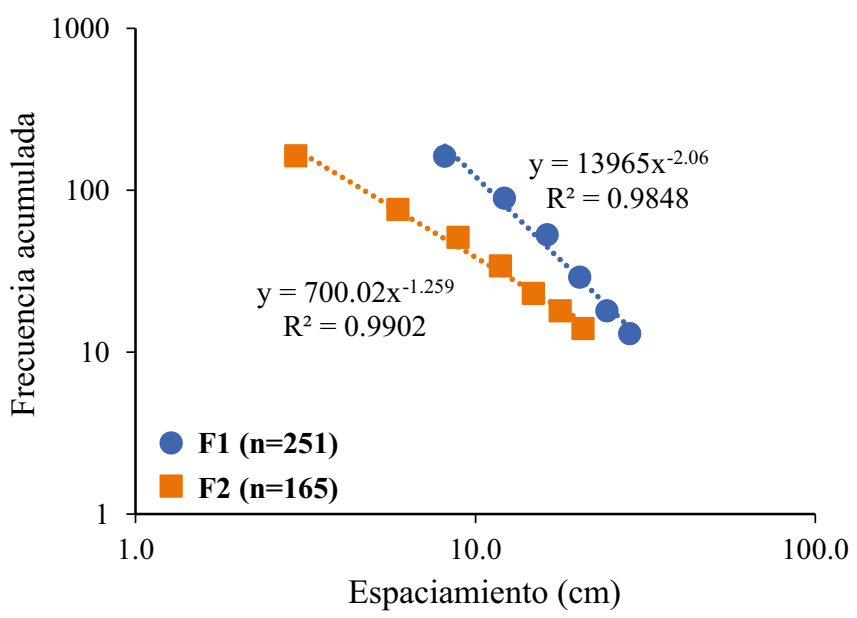

Figura 6. a) Ejemplos de arreglos de fracturas observados en las dos familias de fracturas estudiadas (F1 y F2). b) Gráfico bilogarítmico del tamaño de la apertura contra su frecuencia acumulada para las fracturas F1 y F2. c) Gráfico bilogarítmico del espaciamiento entre fracturas contra su frecuencia acumulada para las fracturas F1 y F2. En los gráficos bilogarítmico se incluye el número de datos (n) que se usó para nuestro análisis. 
Tabla 1. Resultados de los análisis realizados en las fracturas F1 que afectan a rocas andesíticas (NNW-SSE) para cada línea de muestreo (Vs' - Grado de heterogeneidad en la distribución de la deformación; $\mathrm{CV}$ - Coeficiente de variación; $\mathrm{CV}^{*}$ - Coeficiente de variación con un número de datos reducido; Exp-Ly- Exponente de Lyapunov; Dcaja- Dimensión de caja; Dcorr- Dimensión de correlación; Aper. máx.- Apertura máxima).

\begin{tabular}{lccccccccccc}
\hline Clave & $\begin{array}{c}\text { Long. línea } \\
(\mathrm{cm})\end{array}$ & $\begin{array}{c}\text { Núm. } \\
\text { fracturas }\end{array}$ & \%extensión & $V^{\prime}$ & $\mathbf{C V}$ & $\mathbf{C V}^{*}$ & Exp-Ly & Dcorr & $\begin{array}{c}\text { Dcaja } \\
\text { Intensidad } \\
(\text { No/m) }\end{array}$ & $\begin{array}{c}\text { Aper. máx. } \\
(\mathrm{cm})\end{array}$ \\
\hline NS-058 & 134 & 13 & 1.80 & 0.18 & 0.63 & 0.68 & 0.50 & 0.37 & 0.48 & 12 & 0.2 \\
NS-100 & 107 & 11 & 1.70 & 0.28 & 0.85 & 0.93 & 1.75 & 0.45 & 0.34 & 10 & 0.2 \\
NS-156 & 118 & 11 & 2.50 & 0.34 & 0.76 & 0.83 & 2.09 & 0.48 & 0.32 & 10 & 0.6 \\
NS-235 & 110 & 10 & 2.50 & 0.27 & 0.75 & 0.83 & 1.19 & 0.51 & 0.59 & 19 & 0.2 \\
NS-246 & 166 & 23 & 2.80 & 0.18 & 0.81 & 0.85 & 1.18 & 0.45 & 0.63 & 22 & 0.3 \\
NS-435 & 135 & 16 & 2.00 & 0.23 & 0.69 & 0.73 & 1.38 & 0.55 & 0.45 & 15 & 0.3 \\
NS-445 & 151 & 19 & 2.80 & 0.17 & 1.04 & 1.10 & 1.87 & 0.42 & 0.55 & 18 & 0.4 \\
NS-536 & 127 & 27 & 2.60 & 0.15 & 0.59 & 0.61 & 1.27 & 0.63 & 0.72 & 26 & 0.2 \\
NS-557 & 92 & 8 & 2.00 & 0.36 & 0.44 & 0.50 & 0.94 & 0.44 & 0.23 & 7 & 0.7 \\
NS-609 & 216 & 18 & 2.10 & 0.30 & 0.77 & 0.81 & 1.24 & 0.56 & 0.52 & 17 & 0.6 \\
NS-645 & 210 & 23 & 3.50 & 0.21 & 0.73 & 0.76 & 1.38 & 0.57 & 0.61 & 22 & 0.7 \\
NS-648 & 251 & 18 & 2.60 & 0.18 & 0.85 & 0.90 & 1.30 & 0.48 & 0.54 & 17 & 0.6 \\
NS-754 & 125 & 33 & 2.80 & 0.18 & 0.85 & 0.88 & 1.25 & 0.53 & 0.70 & 32 & 0.2 \\
NS-846 & 156 & 23 & 2.70 & 0.24 & 0.70 & 0.73 & 1.19 & 0.53 & 0.64 & 22 & 0.4 \\
Promedio & & & 2.46 & 0.23 & 0.75 & 0.80 & 1.32 & 0.50 & 0.52 & 17.79 & 0.40 \\
\hline
\end{tabular}

de correlación y exponente de Lyapunov, sugieren que sus valores están ligados al arreglo de fracturas y su cantidad, como ha sido reportado en otros trabajos (Vásquez-Serrano et al., 2019). En condiciones de igual tipo de roca y diferentes eventos de deformación, como se observa en este estudio, el arreglo de fracturas y su cantidad dependen en gran medida con el tipo de roca más que con la cantidad de deformación ligada con un evento tectónico en específico.

En lo que se refiere a la deformación por extensión que acomodan las fracturas, es posible observar que las fracturas F1 acomodan una menor cantidad de deformación ( $2.46 \%$ en promedio) en comparación con las fracturas F2 (6.84 \% en promedio) (Tablas 1 y 2 ). Los valores del parámetro $V s^{\prime}$ sugieren que la distribución de la deformación es heterogénea en ambas familias de fracturas (Figura 7). Este comportamiento confirma lo observado en otros trabajos (Putz-Perrier y Sanderson, 2008) y sigue las bases teóricas propuestas para la deformación de la corteza superior terrestre (Segall and Pollard, 1983; Putz-Perrier y Sanderson, 2008). Es relevante aclarar que para este trabajo solo se estimó la deformación por extensión, por lo que no se consideró a la deformación asociada con el desplazamiento paralelo a las fracturas, el cual es una parte importante de la deformación que acomodan las fracturas F1. Será importante en el futuro hacer más estudios cuantitativos de la deformación a escala mesoscópica, para los eventos de deformación cenozoicos que afectan a las rocas volcánicas del centro de México. Más análisis, como el que se presenta en este trabajo, ayudaran a conocer la cantidad de deformación (por extensión y cizalla) que afectan a las rocas volcánicas de la Faja Volcánica Transmexicana y a entender las variaciones espaciales de los distintos eventos de deformación frágil y su relación con el vulcanismo en cuanto a la alineación de estructuras volcánicas, su composición y tipo de actividad volcánica (García-Palomo et al., 2002; Alaniz-Álvarez et al., 1998; Alaniz-Álvarez, et al., 2002a).

\section{Fracturas y su relación con el sistema geotérmico local}

En los flancos sur y norte del lago de Cuitzeo, principalmente en los poblados de San Agustín del Maíz y Araró (fuera del área de estudio), existen manifestaciones hidrotermales asociadas a un sistema geotér-

Tabla 2. Resultados de los análisis realizados en las fracturas F2 que afectan a rocas andesíticas (ENE-WSW) para cada línea de muestreo (Vs' - Grado de heterogeneidad en la distribución de la deformación; CV- Coeficiente de variación; $\mathrm{CV}^{\star}$ - Coeficiente de variación con un número de datos reducido; Exp-Ly- Exponente de Lyapunov; Dcaja- Dimensión de caja; Dcorr- Dimensión de correlación; Aper. máx.- Apertura máxima ).

\begin{tabular}{lccccccccccc}
\hline Clave & $\begin{array}{c}\text { Long. línea } \\
(\mathrm{cm})\end{array}$ & $\begin{array}{c}\text { Núm. } \\
\text { fracturas }\end{array}$ & \%extensión & $V^{\prime}$ & $\mathbf{C V}$ & $\mathbf{C V}^{*}$ & Exp-Ly & Dcorr & $\begin{array}{c}\text { Dcaja } \\
\text { Intensidad } \\
(\text { No/m) }\end{array}$ & $\begin{array}{c}\text { Aper. máx. } \\
(\mathrm{cm})\end{array}$ \\
\hline EW-121 & 201 & 22 & 5.7 & 0.23 & 0.60 & 0.63 & 1.06 & 0.60 & 0.65 & 21 & 1.8 \\
EW-241 & 141 & 25 & 8.2 & 0.25 & 1.18 & 1.23 & 1.57 & 0.39 & 0.56 & 24 & 1.8 \\
EW-248 & 166 & 21 & 5.2 & 0.25 & 0.85 & 0.89 & 1.59 & 0.53 & 0.59 & 20 & 2.3 \\
EW-323 & 138 & 13 & 4.2 & 0.34 & 0.78 & 0.84 & 1.84 & 0.52 & 0.38 & 12 & 1.6 \\
EW-452 & 167 & 9 & 8.9 & 0.34 & 0.78 & 0.87 & 1.51 & 0.47 & 0.32 & 8 & 3 \\
EW-530 & 114 & 24 & 8.1 & 0.21 & 0.64 & 0.67 & 1.1 & 0.60 & 0.66 & 23 & 1.3 \\
EW-533 & 104 & 10 & 7.4 & 0.52 & 0.90 & 0.99 & 1.61 & 0.39 & 0.32 & 9 & 2 \\
EW-632 & 102 & 12 & 8.1 & 0.44 & 0.69 & 0.75 & 1.62 & 0.39 & 0.39 & 11 & 2.4 \\
EW-633 & 122 & 26 & 8.2 & 0.26 & 0.74 & 0.77 & 1.46 & 0.52 & 0.68 & 25 & 1.4 \\
EW-817 & 90 & 11 & 4.4 & 0.48 & 0.91 & 1.00 & 1.27 & 0.36 & 0.38 & 12 & 1 \\
Promedio & & & 6.8 & 0.33 & 0.81 & 0.86 & 1.463 & 0.48 & 0.49 & 16.5 & 1.86 \\
\hline
\end{tabular}


mico de baja entalpia (Vigil-Barrientos et al., 2017; Del Razo González, 2017; Bermejo-Santoyo, 2018; Mazzoldi et al., 2020; Pérez-Martínez et al., 2021). La disposición en el espacio de las manifestaciones hidrotermales, así como la presencia de fracturas rellenas con material precipitado de actividad hidrotermal (Bermejo-Santoyo, 2018), sugiere que el sistema geotérmico está asociado con las estructuras (fallas NNW-SSE y ENE-WSW) desarrolladas por los eventos de deformación frágil: D1 y D2 (Bermejo-Santoyo, 2018; Mazzoldi et al., 2020). Algunos autores han sugerido que la intersección entre estructuras mayores (fallas kilométricas) puede ayudar a explicar la localización de las manifestaciones hidrotermales (Figura 2. Mazzoldi et al., 2020); aunque también es posible que las fallas ENE-WSW (evento D2) jueguen un papel importante en el flujo de fluidos del sistema geotérmico, debido a la orientación $\sim \mathrm{E}-\mathrm{W}$ que tienen las manifestaciones hidrotermales en el flanco sur del lago de Cuitzeo (Bermejo-Santoyo, 2018; Mazzoldi et al., 2020).

Sumado a los estudios tectónicos, estructurales y estratigráficos realizados en los últimos años en el área del lago de Cuitzeo (TrujilloHernández, 2017; Bermejo-Santoyo, 2018; Mazzoldi et al., 2020), nuestros resultados sugieren que las rocas andesíticas de la secuencia Tarímbaro, la cual tiene aproximadamente $2 \mathrm{~km}$ de espesor y se encuentra a $\sim 1 \mathrm{~km}$ de profundidad en la zona del lago de Cuitzeo (Mazzoldi et al., 2020), tienen el potencial para formar parte de la capa permeable del sistema geotérmico de Cuitzeo (reservorio). La intensidad de fracturas estimada en este trabajo sugiere que los dos eventos de deformación contribuyen con una cantidad de fracturas similar, a pesar de que son producto de una cantidad de extensión diferente (Tablas 1 y 2; Figura 8). Sumado a las fracturas F1 y F2, se encuentran las fracturas laminadas que fueron reactivadas durante el evento de deformación frágil D2. La presencia de las tres familias de fracturas (laminadas, F1 y F2) genera una porosidad secundaria importante (porosidad de fracturas), como lo sugieren los datos de dimensión de caja estimados para cada familia de fractura. Este parámetro fractal suele tener una relación lineal positiva con la porosidad, como lo sugiere Miranda-Martínez et al. (2006), e incrementa su valor con la presencia de más de una familia de fracturas en las rocas (Barton y LaPointe, 1995). Asimismo, el arreglo de las fracturas determinado para las rocas en estudio muestra que las fracturas F1 y F2 tienden a agruparse. Este patrón contribuye a un aumento significativo de la porosidad y permeabilidad en los sitios donde se cruzan los dos grupos de fracturas. Por otro lado, la distribución heterogénea de la deformación está ligada a variaciones en la apertura de las fracturas y a la deformación localizada. Esta última, está relacionada con la presencia de grupos de fracturas y con fracturas que tienen una apertura grande a escala mesoscópica (Figura 7, Tablas 1 y 2). El grado de heterogeneidad en la deformación observado en las fracturas F2 es congruente con las aperturas máximas estimadas para a)

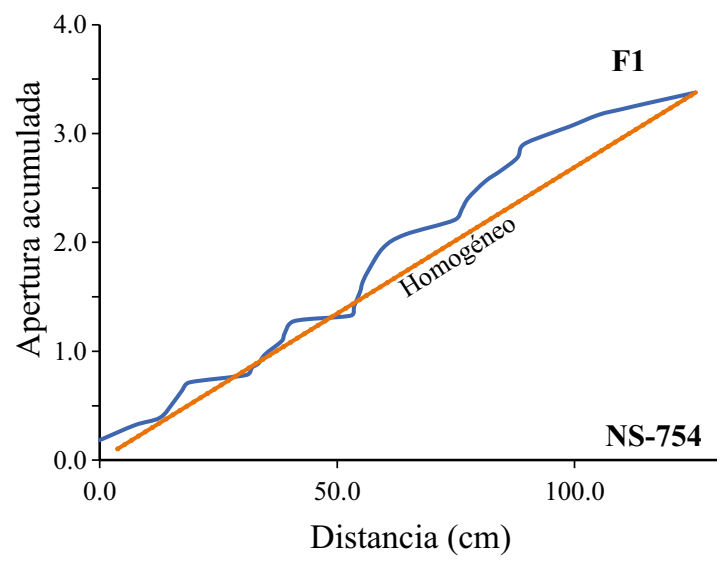

c)

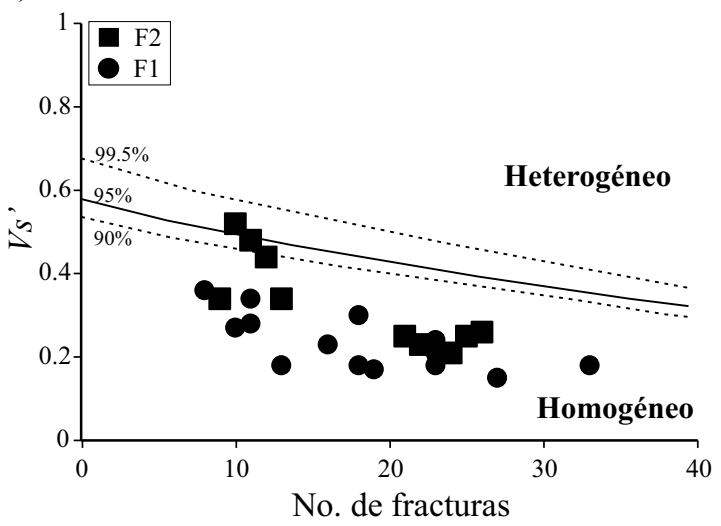

b)

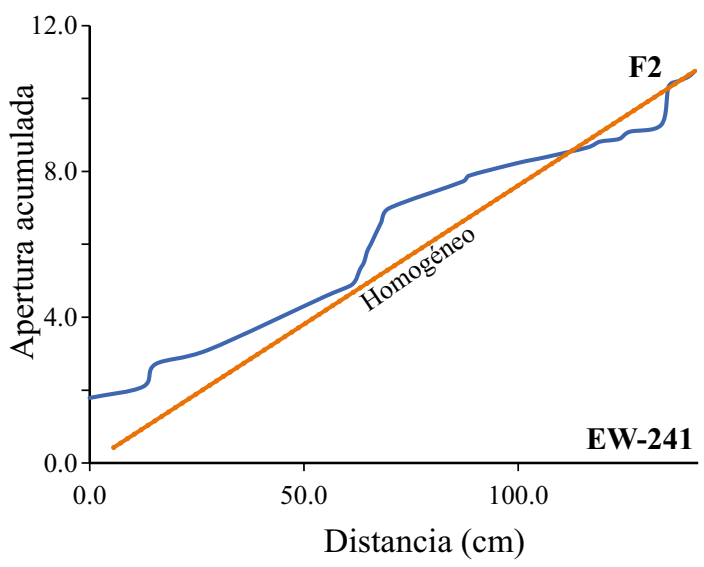

d)

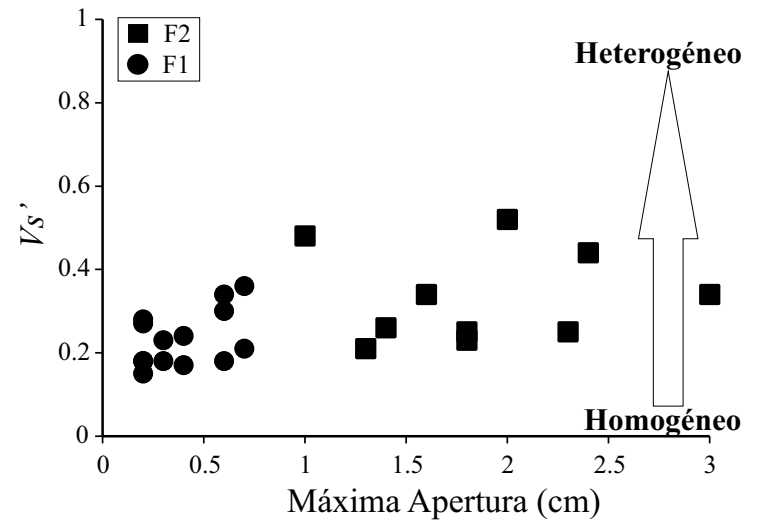

Figura 7. a) y b) Ejemplos de dos gráficos de apertura acumulada contra la distancia para las familias de fracturas F1 y F2, y su comparación con una distribución homogénea en la deformación. c) Gráfico del grado de heterogeneidad en la distribución de la deformación ( $\left.V s^{\prime}\right)$ contra el número de fracturas observado en cada línea de muestreo. c) Gráfico de $V s^{\prime}$ contra la apertura máxima medida en cada familia de fracturas (F1 y F2) para cada línea de muestreo. Nótese los diferentes grados de heterogeneidad en la distribución de la deformación que acomodan las fracturas F1 y F2. 

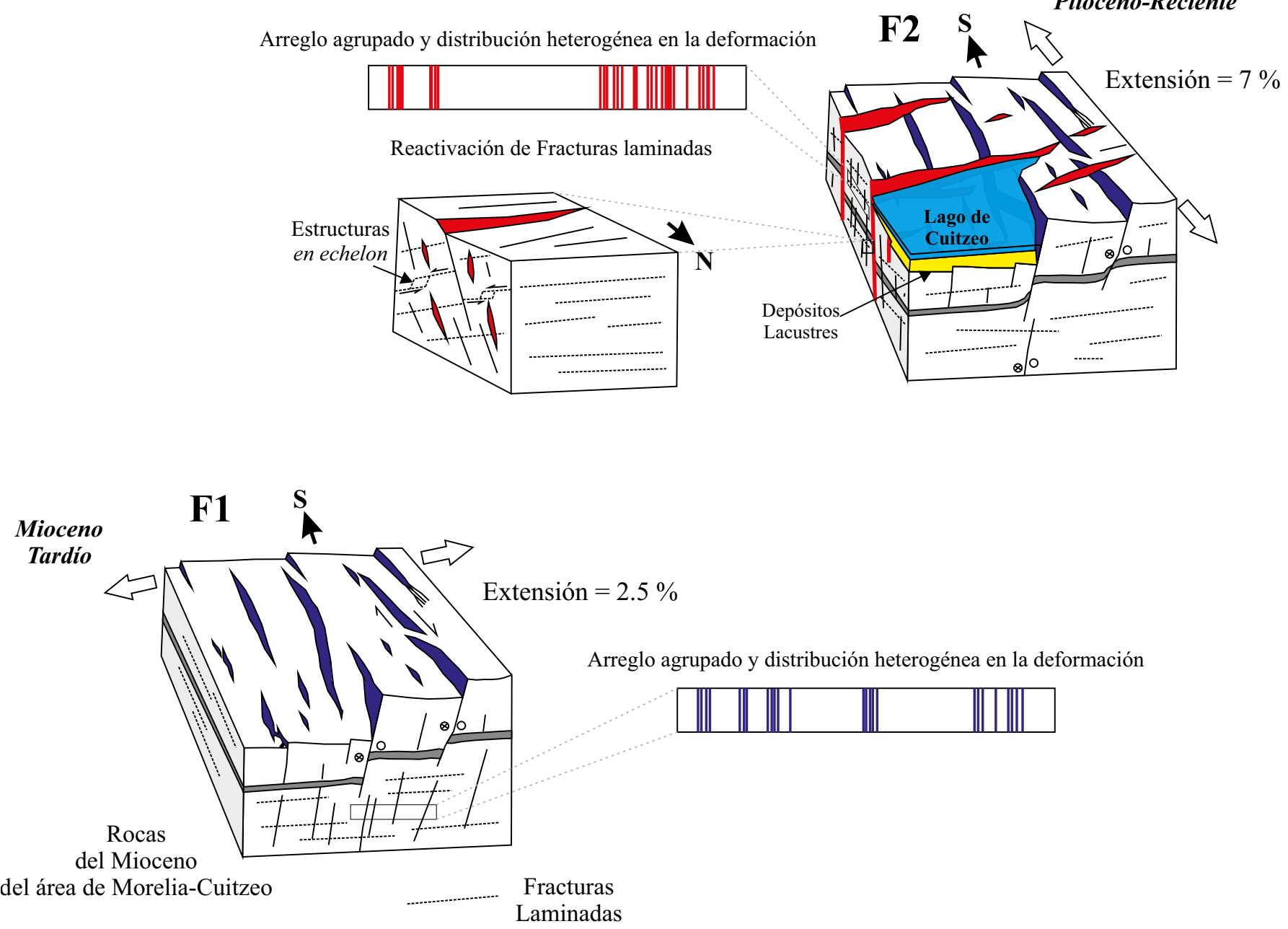

Figura 8. Modelos esquemáticos de la formación, arreglo de fracturas y distribución de la deformación en rocas andesíticas del Mioceno dentro de la región MoreliaCuitzeo. Las fracturas F1 se relacionan con la actividad de fallas laterales derechas con componente normal que tienen rumbo NNW-SSW y forman un arreglo de fracturas agrupadas a escala mesoscópica. La deformación por extensión que acomodan las fracturas F1 es de 2.5\%. Por otro lado, las fracturas F2 están asociadas con fallas normales (ENE-WSW), forman fracturas agrupadas y acomodan una extensión $\sim \mathrm{N}-\mathrm{S}$ de $\sim 7 \%$. En ambas familias de fracturas la deformación quebradiza se distribuye de manera heterogénea en las rocas que afectan.

estas fracturas, y sugiere que estas estructuras tienen una mayor contribución en la generación de porosidad secundaria debido a que tienen una mayor apertura en comparación con las fracturas F1 (Figuras 6 y 7). Finalmente, la reactivación de las fracturas laminadas aumenta la porosidad de manera significativa, sobre todo por la abundante presencia de estructuras en echelon a escala mesoscópica (Figura 5).

Todas las características anteriores, demuestran el potencial que tienen las rocas andesíticas de la secuencia Tarímbaro de formar parte de la capa permeable del sistema geotérmico de la zona del lago de Cuitzeo.

\section{CONCLUSIONES}

Las familias de fracturas estudiadas en este trabajo están asociadas con dos eventos de deformación (D1 y D2) ocurridos entre el Mioceno y el reciente. Las fracturas $\mathrm{F} 1$ del evento $\mathrm{D} 1$ son principalmente híbridas y acomodaron una extensión de $\sim 2.5 \%$, mientras que las fracturas F2 están representadas por fracturas extensionales que acomodan una extensión de $\sim 7 \%$. Ambas familias de fracturas tienen un arreglo agrupado y una intensidad de fracturas similar ( 17 fracturas/metro).
Con base en nuestros resultados, proponemos que los patrones de espaciamiento y apertura están ligados a los eventos de deformación que forman a cada una de las familias de fracturas, mientras que el arreglo y la cantidad de las fracturas son variables ligadas al tipo de roca más que a la cantidad de deformación. El desarrollo de las fracturas F2 ocurre en conjunto con la reactivación de fracturas laminadas previas. Esta reactivación se acomoda mediante deformación por cizalla y estructuras en echelon. La presencia de fracturas abiertas, su cantidad y arreglo, así como la distribución heterogénea en la deformación frágil, sugieren que las rocas de la secuencia Tarímbaro tienen el potencial de ser la capa permeable del sistema geotérmico de la zona del lago de Cuitzeo.

\section{AGRADECIMIENTOS}

Los autores de este manuscrito agradecen la valiosa contribución del Dr. Víctor Hugo Garduño Monroy, valoramos y atesoramos mucho sus enseñanzas, y admiramos el entusiasmo y la pasión que ponía en su trabajo. Este trabajo fue financiado por el proyecto CeMIE-Geo P-17 que estuvo a cargo del Dr. Garduño. Así mismo, agradecemos 
los valiosos comentarios y correcciones al manuscrito por parte del Dr. Luis Delgado-Argote, el Dr. Pierre Lacan, y los editores Dra. Isabel Israde y Dr. José Luis Macías.

\section{REFERENCIAS}

Alaniz-Álvarez, S.A., Nieto-Samaniego, A.F., Ferrari, L., 1998, Effect of the strain rate in the distribution of monogenetic and polygenetic volcanism in the Transmexican Volcanic Belt: Geology, 26, 591-594.

Alaniz-Álvarez, S. A., Nieto-Samaniego, A. F., Morán-Zenteno, D. J., AlbaAldave, L., 2002a, Rhyolitic volcanism in extension zone associated with strike-slip tectonics in the Taxco region, southern Mexico: Journal of Volcanology and Geothermal Research, 118(1-2), 1-14.

Alaniz-Álvarez, S.A., Nieto-Samaniego, A.F., Orozco-Esquivel, M.T., VasalloMorales, L.F., Xu, S.S., 2002b, El Sistema de Fallas Taxco- San Miguel de Allende: Implicaciones en la deformación post-Eocénica del centro de México: Boletín de la Sociedad Geológica Mexicana, 55, 12-29.

Alaniz-Álvarez, S. A., Nieto-Samaniego, Á. F., 2005, El sistema de fallas TaxcoSan Miguel de Allende y la Faja Volcánica Transmexicana, dos fronteras tectónicas del centro de México activas durante el Cenozoico: Boletín de la Sociedad Geológica Mexicana, 57(1), 65-82.

Arce, J. L., Layer P. W., Morales-Casique, E., Benowitz, J. A., Rangel, E., Escolero, O. A., 2013, New constraints on the subsurface geology of the Mexico City Basin: The San Lorenzo Tezonco deep well, on the basis of 40Ar/39Ar geochronology and whole-rock chemistry: Journal of Volcanology and Geothermal Research, 266, 34-49.

Astíz-Delgado, L.M., 1980, Sismicidad en Acambay, Estado de México, El temblor del 22 de febrero de 1979:Ciudad de México, México, Universidad Nacional Autónoma de México, Facultad de Ingeniería, Tesis Doctoral, $130 \mathrm{pp}$.

Avellán, D.R., Cisneros-Máximo, G., Macías, J.L., Gómez-Vasconcelos, M.G., Layer, P.W., Sosa-Ceballos, G., Robles-Camacho, J., 2020, Eruptive chronology of monogenetic volcanoes northwestern of Morelia-Insights into volcano-tectonic interactions in the central-eastern MichoacánGuanajuato Volcanic Field, México: Journal of South American Earth Sciences, 100, 102554.

Barton, C.C., LaPointe, P.R., 1995, Fractal Analysis of Scaling and Spatial Clustering of Fractures, in Barton, C., La Pointe, P.R. (eds.), Fractals in Earth Science: New York, Plenum Press, 141-178.

Bermejo Santoyo, G., 2018, Análisis geométrico y fractal de fracturas geológicas en los campos geotérmicos del Lago de Cuitzeo: Morelia, Michoacán, México, Universidad Michoacana de San Nicolás Hidalgo, tesis de maestría en ciencias, $132 \mathrm{pp}$.

Billi, A., Salvini, F., Storti, F., 2003, The damage zone-fault core transition in carbonate rocks: implications for fault growth, structure and permeability: Journal of Structural Geology, 25, 1779-1794.

Cladouhos T.T., Marrett, R., 1996, Are fault growth and linkage models consistent with power-law distributions of fault lengths?: Journal of Structural Geology, 18, 281-293.

Cox, D.R., Lewis, P.A.W., 1966, The statistical analysis of series of events: London, Methuen's, Monographs on Applied Probability and Statistics, $285 \mathrm{pp}$.

Davy, P., Sornette, A., Sornette, D., 1990, Some consequences of a proposed fractal nature of continental faulting: Nature, 348, 56-58.

Del Razo González, A., 2017, Análisis gravimétrico y geológico para definir el modelo conceptual del yacimiento geotérmico de San Agustín del Maíz, Michoacán, México: Ciudad de México, México, Instituto Politécnico Nacional, Tesis de Licenciatura en Ingeniería Geofísica, 98 pp.

Evans, J.P., Forster, C.B., Goddard, J.V., 1997, Permeability of fault-related rocks, and implications for hydraulic structure of fault zones: Journal of Structural Geology, 19, 1393-1404.

Ferrari, L., 2000, Avances en el conocimiento de la Faja Volcánica Transmexicana durante la última década: Boletín de la Sociedad Geológica Mexicana, 53(1), 84-92.

Ferrari, L., Orozco-Esquivel, T., Manea, V., Manea, M., 2011, The dynamic history of the Trans-Mexican Volcanic Belt and the Mexico subduction zone: Tectonophysics, 522-523, 122-149.
Fossen, H., 2010, Structural geology: New York, Cambridge University Press, First Edition, $481 \mathrm{pp}$.

García-Palomo, A., Macías, J.L., Arce, J.L., Capra, L., Garduño, V.H., Espíndola J.M., 2002, Geology of the Nevado de Toluca volcano and surrounding areas, Central Mexico: Boulder, Colorado, Geological Society of America Map and Chart Series MCH089, 26 pp.

García-Palomo, A., Macías, J.L., Garduño, V.H., 2000, Miocene to Recent structural evolution of the Nevado de Toluca volcano region, Central Mexico: Tectonophysics, 318, 281-302.

Garduño-Monroy, V.H., Pérez-Lopez, R., Israde-Alcantara, I., RodríguezPascua, M.A., Szynkaruk, E., Hernández-Madrigal, V.M., García-Zepeda, M.L., Corona-Chávez, P., Ostroumov, M., Medina-Vega1, V.H., GarcíaEstrada, G., Carranza, O., Lopez-Granados, E., Mora Chaparro, J.C., 2009, Paleoseismology of the southwestern Morelia-Acambay fault system, central Mexico: Geofísica Internacional, 48(3), 319-335.

Gillespie, P.A., Walsh, J.J., Watterson, J., Bonson, C.G., Manzocchi, T., 2001, Scaling relationships of joint and vein arrays from The Burren, Co. Clare, Ireland: Journal of Structural Geology, 23(2-3), 183-201.

Gómez-Tuena, A., Carrasco-Núñez, G., 2000, Cerro Grande volcano: the evolution of a Miocene stratocone in the early Trans-Mexican Volcanic Belt: Tectonophysics, 318(1-4), 249-280.

Gómez-Vasconcelos, M.G., Garduño-Monroy, V.H., Macías, J.L., Layer, P.W., Benowitz, J.A., 2015, The Sierra de Mil Cumbres, Michoacán, México: Transitional volcanism between the Sierra Madre Occidental and the Trans-Mexican Volcanic Belt: Journal of Volcanology and Geothermal Research, 301, 128-147.

Gómez-Vasconcelos, M.G., Macías, J.L., Avellán, D.R., Sosa-Ceballos, G., Garduño-Monroy, V.H., Cisneros-Máximo, G., Layer., P.W., Benowitz, J., López-Loera, H., Mendiola-López, F., Perton, M., 2020, The control of preexisting faults on the distribution, morphology, and volume of monogenetic volcanism in the Michoacán-Guanajuato Volcanic Field: The Geological Society of America Bulletin, 132(11-12), 2455-2474.

Henry, C.D., Aranda-Gómez, J.J. 1992, The real southern Basin and Range: Mid-to late Cenozoic extension in Mexico: Geology, 20(8), 701-704.

Hooker, J.N., Laubach, S.E., Marrett, R., 2013, Fracture-aperture sized frequency, spatial distribution, and growth processes in strata-bounded and nonstratabounded fractures, Cambrian Meson Group, NW Argentina: Journal of Structural Geology, 54, 54-71.

Hooker, J.N., Laubach, S.E., Marrett, R., 2018, Microfracture spacing distributions and the evolution of fracture patterns in sandstones: Journal of Structural Geology, 108, 66-79.

Kuiper, N.H., 1960, Tests concerning random points on a circle: Nederlandes Akademie van Wetenschappen Proceedings, A36, 38-47.

La Pointe, P.R., Hudson, J.A., 1985, Characterization and interpretation of rock mass joint patterns: Boulder, Colorado, United States of America. The Geological Society of America, 199, 37 pp.

Lacan, P., Ortuño, M., Audin, L., Perea, H., Baize, S., Aguirre-Díaz, G., Zúñiga, F. R., 2018, Sedimentary evidence of historical and prehistorical earthquakes along the Venta de Bravo Fault System, Acambay Graben (Central Mexico): Sedimentary Geology, 365, 62-77.

Langridge, R.M., Weldon, R.J., Moya, J.C., Suárez, G., 2000, Paleoseismology of the 1912 Acambay earthquake and the Acambay-Tixmadejé fault, TransMexican Volcanic Belt: Journal of Geophysical Research, 105, 3019-3037.

Langridge, R.M., Persaud, M., Zúñiga, F.R., Aguirre-Díaz, G.J., Villamor, P., Lacan, P., 2013, Preliminary paleoseismic results from the Pastores fault and its role in the seismic hazard of the Acambay graben, Trans-Mexican Volcanic Belt, Mexico: Revista Mexicana de Ciencias Geológicas, 30, 463-481.

Laubach, S.E., Lamarche, J., Gauthier, B.D.M., Dunne, W.M., Sanderson, D.J., 2018, Spatial arrangement of faults and opening-mode fractures: Journal of Structural Geology, 108, 2-15.

Magnall, 2018, Commonalities in the emplacement of cooling-limited lavas: insights from the 2011-2012 Cordón Caulle rhyolitic eruption: Lancaster University, Tesis de doctorado, $332 \mathrm{pp}$.

Mandelbrot, B.B., 1967, How long is the coast of Britain? Statistical self-similarity and fractional dimensión: Science, 156, 636-638.

Manea, V.C., Manea, M., Ferrari, L., 2013, A geodynamical perspective on the subduction of Cocos and Rivera plates beneath Mexico and Central America: Tectonophysics, 609, 56-81. 
Marrett, R., Ortega, O.J., Kelsey, C.M., 1999, Extent of power-law scaling for natural fractures in rock: Geology, 27, 799-802.

Martínez-Reyes, J., Nieto-Samaniego, Á.F., 1990, Efectos geológicos de la tectónica reciente en la parte central de México: Revista Mexicana de Ciencias Geológicas, 9(1), 33-50.

Mazzoldi, A., GaRduño-Monroy, V.H., Gómez Cortes, J.J., Guevara Alday, J.A., 2020, Geophysics for geothermal exploration. Directional-derivativesbased computational filters applied to geomagnetic data at lake Cuitzeo, Mexico: Geofísica Internacional, 59(2), 105-135.

Mennella, L., Garduño-Monroy, V.H., Bonassi, O., 2000, Fault-slip analysis in the basal units of the Mexican Volcanic Belt on the eastern flank of the Tzitzio Anticline, Michoacan Mexico: GSA Special Paper, 334, 237-246.

Miranda-Martínez, M.E., Oleschko K., Parrot J.F., Castrejon-Vacio, F., Taud, H., Brambila-Paz, F., 2006, Porosidad de los yacimientos naturalmente fracturados: una clasificación fractal: Revista Mexicana de Ciencias Geológicas, 23(2), 199-214.

Nieto-Samaniego, A.F., Alaniz-Álvarez, S., Tolson, G., Xu, S., Pérez-Venzor, A., 2003, Estimación de densidades, distribuciones de longitud y longitud total de fracturas: un caso de estudio en la Falla de Los Planes, La Paz, B.C.S. Boletín de la Sociedad Geológica Mexicana, LVI (1), 1-9.

Nieto-Sanamiego, A.F., Alaniz-Álvarez, S., Tolson, G., Oleschko, K., Korvin. G., Xu, S.S., Pérez-Venzor, J.A., 2005, Spatial Distribution, Scaling and Selfsimilar Behavior of Fracture Arrays in the Los Planes Fault, Baja California Sur, México: Pure and Applied Geophysics, 162, 805-826.

Ortuño, M., Zúñiga, F.R., Aguirre-Díaz, G.J., Carreón-Freyre, D., Cerca, M., Roverato, M., 2015, Holocene paleo-earthquakes recorded at the transfer zone of two major faults: the Pastores and Venta de Bravo faults (TransMexican Volcanic Belt): Geosphere 11, 160-184.

Ortuño, M., Coromitas, O., Zúñiga, F.R., Lacan, P., Perea, H., Aguirre-Díaz, G., Štěpančíková, P., Villamor, P., Ramirez-Herrera, T., 2018, Evidence of recent ruptures in the central faults of the Acambay Graben (Central Mexico): Sedimentary Geology, 326, 17-37, https://doi.org/10.1016/j. geomorph.2018.07.010

Pardo, M., Suárez, G., 1995, Shape of the subducted Rivera and Cocos plates in southern Mexico: Seismic and tectonic implications: Journal of Geophysical Research, 100, 12357-12373.

Pardo, M., Suárez, G., 1993, Steeps subduction geometry of the Rivera plate beneath the Jalisco block in western Mexico: Geophysical Research Letters, 20, 2391-2394.

Pasquarè, G., Ferrari, L., Garduño-Monroy, V.H., Tibaldi, A., Vezzoli, L., 1991, Geologic map of the central sector of the Mexican Volcanic Belt, states of Guanajuato and Michoacán, Mexico: Boulder, CO, The Geological Society of America, Map and chart series, MCH072, 1 mapa con texto, 22 pp.

Pérez-Martínez, I., Villanueva-Estrada, R.E., Rodríguez-Díaz, A.A., Canet, C., García, R., Ramos-Leal, A., Cambrón, F., 2021, Diffuse gases in soil of Araro-Simirao geothermal system, Michoacan, Mexico: Andean Geology, 48(3), 496-513, doi:http://dx.doi.org/10.5027/andgeoV48n3-3327.

Pola, A., Martínez-Martínez, J., Macías, J.L., Fusi, N., Crosta, G., GarduñoMonroy, V.H., Núñez-Hurtado, J.A., 2016, Geomechanical characterization of the Miocene Cuitzeo ignimbrites, Michoacán, Central Mexico: Engineering Geology, 214, 79-93.

Putz-Perrier, M.W., Sanderson, D.J., 2008, Spatial distribution of brittle strain in layered sequences: Journal of Structural Geology, 30(1), 50-64.

Ramsay, J.G., 1967, Folding and Fracturing of Rocks: New York, McGraw-Hill, $568 \mathrm{pp}$.

Ramsey, J.M., Chester, F.M., 2004, Hybrid fracture and the transition from extension fracture to shear fracture: Nature, 428(6978), 63-66.

Riley, P., Tikoff, B., Murray, A.B., 2011, Quantification of fracture networks in non-layered, massive rock using synthetic and natural data sets: Tectonophysics, 505, 44-56.

Rodríguez-Pascua, M.A., Pérez-López, R., Garduño-Monroy, V.H., Perucha, M.A., 2017, Estimation of the epicentral area of the 1912 Acambay earthquake (M6.9, Mexico) determined from the earthquake archaeological effects (EAE) and the ESI07 macroseismic scale: Quaternary International, $451,74-86$

Rosenstein, M.T., Collins, J.J., De Luca, C.J., 1993, A practical method for calculating largest Lyapunov exponents from small data sets. Physica D: Nonlinear Phenomena, 65, 117-134.
Segall, P., Pollard, D.D., 1983, Nucleation and growth of strike slip faults in granite: Journal of Geophysical Research: Solid Earth, 88(B1), 555-568.

Silva-Mora, L., 1995, Hoja Morelia 14Q-9(2) con resumen de la geología de la hoja Morelia, estados de Michoacán y Guanajuato: México, D.F., Universidad Nacional Autónoma de México, Instituto de Geología, Cartas Geológicas de México, serie de, 1(100.000), 1 mapa.

Soria-Caballero, D. C., Garduño-Monroy, V. H., Alcalá, M., Velázquez-Bucio, M. M., Grassi, L., 2019. Evidence for quaternary seismic activity of the La Alberca-Teremendo fault, Morelia region, Trans-Mexican Volcanic Belt: Revista Mexicana de Ciencias Geológicas, 36(2), 242-258.

Sunyé-Puchol, I., Lacan, P., Ortuño,M., Villamor, P., Audin, L., Zúñiga, F.R., Langridge, R.M., Aguirre-Díaz, G.J., Lawton, T.F., 2015, La falla San Mateo: Nuevas evidencias paleosismológicas de fallamiento activo en el graben de Acambay, México: Revista Mexicana de Ciencias Geológicas, 32, 361-375.

Suter, M., 2015, The 1567 MW7.2 Ameca, Jalisco earthquake (western TransMexican Volcanic Belt): Surface rupture parameters, seismogeological effects, and macroseismic intensities from historical sources: Seismological Society of America Bulletin, 105, 646-656.

Suter, M., Quintero, O., Johnson, C.A., 1992, Active faults and state of stress in the central part of the Trans-Mexican Volcanic Belt, Mexico 1. The Venta de Bravo Fault: Journal of Geophysical Research, 97, 11983-11994.

Suter, M., Carrillo-Martínez, M., López-Martínez, M., Farrar, E., 1995a, The Aljibes half-graben: Active extension at the boundary between the transMexican volcanic belt and the Basin and Range Province, Mexico: GSA Bulletin, 107(6), 627-641.

Suter, M., Quintero-Legorreta, O., López-Martínez, M., Aguirre-Díaz, G., Farrar, E., 1995b, The Acambay graben: Active intraarc extensión in the TransMexican volcanic belt, Mexico: Tectonics, 14(6), 1245-1262, https://doi. org/10.1029/95TC01930

Suter, M., Carrillo-Martínez, M., Quintero-Legorreta, O., 1996, Macroseismic Study of Shallow Earthquakes inMacroseismic Study of Shallow Earthquakes in the Central and Eastern Parts of the Trans-Mexican Volcanic Belt, Mexico: Bulletin, Seismological Society America, 86(6), 1952-1963.

Trujillo-Hernández, N., 2017. Estudio geológico, geoquímico y mineralógico de las secuencias volcánicas de la porción suroeste del Lago de Cuitzeo, Michoacán, ligadas a la zona geotérmica de San Agustín del Maíz: Morelia, Michoacán, México, Universidad Michoacana de San Nicolas de Hidalgo, Tesis de Maestría, 83 pp.

Urbina, F., Camacho, H., 1913, La zona megaséismica Acambay-Tixmadeje, estado de México: conmovida el 19 de noviembre de 1912 (No. 32): Ciudad de México, México, Imprenta y fototipia de la Secretaría de Fomento, $125 \mathrm{pp}$.

Vásquez-Serrano, A., 2013, Análisis fractal de fracturas geológicas en un análogo expuesto de yacimientos carbonatados naturalmente fracturados y su aplicación en modelos de flujo de fluidos: Ciudad de México, México, Universidad Nacional Autónoma de México, Tesis de Maestría, 153 pp.

Vásquez-Serrano, A., 2020, Frackita: A MATLAB code for the 1D analysis of geological fractures, en Reunión Anual de la Unión Geofísica Mexicana: Guadalajara, México, GEOS, 40(1), resumen GET-9, 41.

Vásquez-Serrano, A., Camacho-Rangel, R., Arce-Saldaña, J.L., Morales-Casique, E., 2019, Análisis de fracturas geológicas en el pozo Agrícola Oriental 2C, Ciudad de México y su relación con fallas mayores: Revista Mexicana de Ciencias Geológicas, 36(1), 38-53.

Vigil-Barrientos, A.J., 2017, Caracterización geológica y geoquímica de fluidos en el sistema geotérmico Araró, Michoacán, México: San Nicolas de los Garza, Nuevo León, México, Universidad Autónoma de Nuevo León, Tesis Doctoral, $102 \mathrm{pp}$.

Wang, Q., Laubach, S.E., Gale, J.F.W., Ramos, M.J., 2019, Quantified fracture (joint) clustering in Archean basement, Wyoming: application of the normalized correlation count method: Petroleum Geoscience, 25(4), 415-428.

Manuscrito recibido: febrero 15, 2021

Manuscrito corregido recibido: septiembre 13, 2021

Manuscrito aceptado: septiembre 21, 2021 